\title{
Nigeria: Third Review Under the Policy Support Instrument-Staff Report; Staff Statement; Press Release on the Executive Board Discussion; and Statement by the Executive Director for Nigeria
}

In the context of the third review under the Policy Support Instrument for Nigeria, the following documents have been released and are included in this package:

- $\quad$ The staff report for the Third Review Under the Policy Support Instrument, prepared by a staff team of the IMF, following discussions that ended on February 27, 2007, with the officials of Nigeria on economic developments and policies. Based on information available at the time of these discussions, the staff report was completed on June 13, 2007. The views expressed in the staff report are those of the staff team and do not necessarily reflect the views of the Executive Board of the IMF.

- A staff statement of June 27, 2007 updating information on recent developments.

- $\quad$ A Press Release summarizing the views of the Executive Board as expressed during its June 27, 2007 discussion of the staff report that completed the review.

- $\quad$ A statement by the Executive Director for Nigeria.

The documents listed below have been or will be separately released.

Joint Staff Assessment of the Poverty Reduction Strategy Paper Progress

Report-National Economic Empowerment and Development Strategy

Letter of Intent sent to the IMF by the authorities of Nigeria*

Poverty Reduction Strategy Paper Progress Report—National Economic

Empowerment and Development Strategy

Technical Memorandum of Understanding*

Policy Statement by the Government of Nigeria on the Fourth Phase of the

Nigerian Economic Reform Program Under the Policy Support Instrument*

*Also included in Staff Report

The policy of publication of staff reports and other documents allows for the deletion of market-sensitive information.

To assist the IMF in evaluating the publication policy, reader comments are invited and may be sent by e-mail to publicationpolicy@imf.org.

Copies of this report are available to the public from

International Monetary Fund • Publication Services

$70019^{\text {th }}$ Street, N.W. • Washington, D.C. 20431

Telephone: (202) 623-7430 • Telefax: (202) 623-7201

E-mail: publications@imf.org • Internet: http://www.imf.org

Price: $\$ 18.00$ a copy

International Monetary Fund

Washington, D.C. 



\section{INTERNATIONAL MONETARY FUND}

\section{NIGERIA}

\section{Third Review Under the Policy Support Instrument}

Prepared by the African and Policy Development and Review Departments (In consultation with other departments)

Approved by Siddharth Tiwari and Carlo Cottarelli

June 13, 2007 


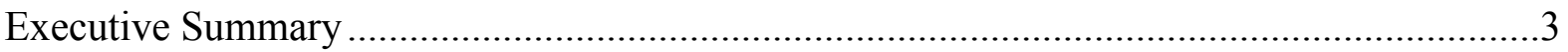

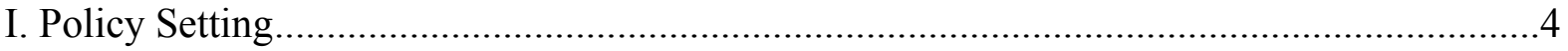

II. Maintaining Macroeconomic Stability ..........................................................................

A. Managing Fiscal Developments..........................................................................

B. Strengthening Monetary Policy Implementation ………........................................

III. Safeguarding Wealth Through Asset and Liability Management.....................................11

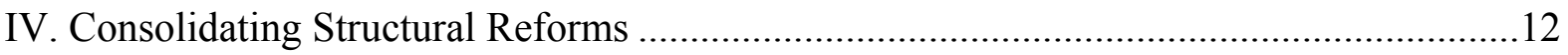

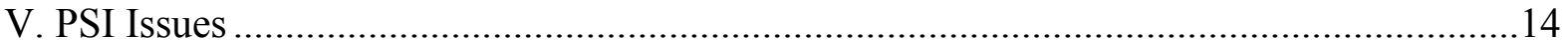

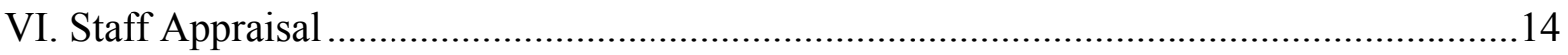

Tables

1. Selected Economic Indicators 2004-09 ………….....................................................16

2a. Consolidated Government (Cash Basis), 2004-09 ….........................................................17

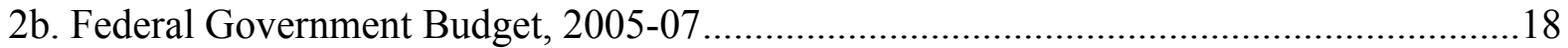

2c. Consolidated and Federal Government, 2004-09.........................................................19

3a Central Bank of Nigeria (CBN) Analytical Quarterly Balance Sheet, 2004-07 ................20

3b. Monetary Survey, 2004-07 .......................................................................................2

4. Balance of Payments, 2004-10 ……………………..................................................22

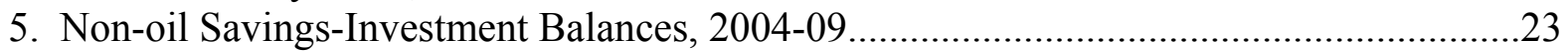

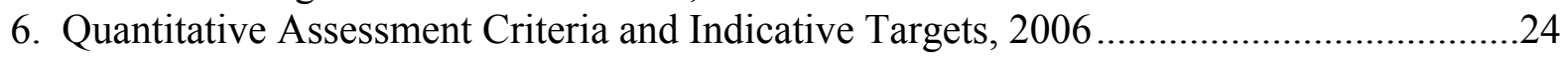

7. Selected Millennium Development Goals .....................................................................25

8a. Structural Assessment Criteria for the 3rd Review, and Structural Benchmarks

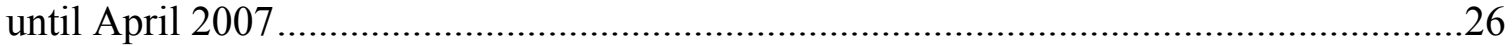

8b. Structural Assessment Criteria for the 4th Review and Structural Benchmarks

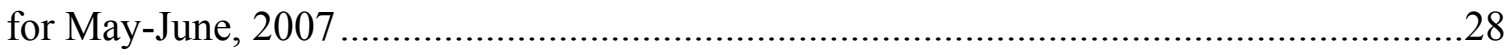

Boxes

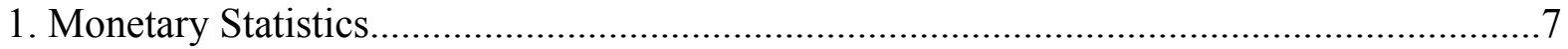

2. Key Issues Raised by the Workshop on Oil Savings and the Infrastructure Gap................11

Appendix

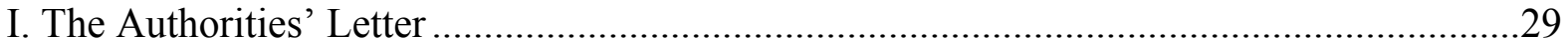

Attachment I. Policy Statement ....................................................................................

Attachment II. Technical Memorandum of Understanding (TMU) ....................................43

Annexes

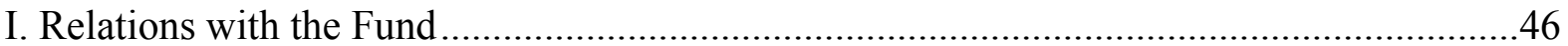

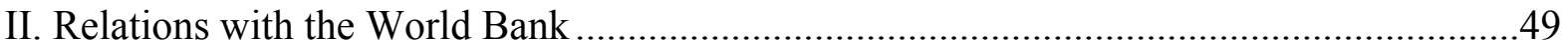

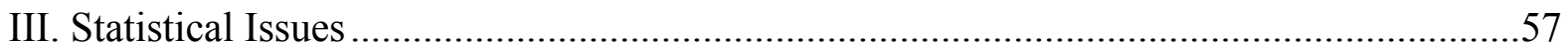




\section{EXECUTIVE SUMMARY}

Ambitious structural reforms undertaken by the outgoing Obasanjo administration have resulted in a stronger policy framework as well as a strengthened macroeconomic position. Robust growth, lower inflation, a dramatic decline in debt, and an accumulation of significant reserves are among the notable economic achievements. It will be important to consolidate and institutionalize these gains as the foundations for sustained and improved economic performance.

The ongoing reforms boosted confidence and sustained strong performance but policy implementation weakened in 2006. Non-oil growth accelerated and its prospects are promising. Inflation remained in line with the single-digit program target owing to a likely increase in confidence and money demand (reflecting, inter alia, the successful consolidation of the banking sector). Nevertheless, growth of the monetary aggregates picked up and a one-time increase in spending by the states in late 2006 meant that the fiscal stance and monetary policy were more expansionary than programmed. Following a strong record of implementing the ambitious structural reform agenda in the first two reviews (only one technical waiver was requested), performance for this review was weaker. Waivers are requested in several cases. The staff is supportive because reforms across most areas are well advanced or have been completed albeit with delay.

The challenge over the recent past and the immediate future is managing the economy and the policy framework through the political transition. Fiscal and monetary policy need to remain consistent with macroeconomic stability. Preliminary indications are that policy implementation has improved in 2007 including through the successful resistance of intense pressures to spend oil savings in the run up to the April elections. For the new government, reaffirming the political agreement on the use of oil revenues will be important in this regard. Guidelines to ensure the effective use of oil savings and good quality of capital spending need to be developed and implemented and structural reforms need to be taken forward. Failure to do so could jeopardize the macroeconomic stability and growth potential_ important elements in Nigeria's fight against poverty.

The staff recommends completion of the third review including to support policy continuity through the political transition. The staff proposes waivers for two missed quantitative and five structural assessment criteria, and modification of the end-June 2007 monetary targets. 


\section{Policy Setting ${ }^{1}$}

1. The accomplishments of the outgoing Obasanjo administration include improved macroeconomic stability and a better policy framework. Robust growth, lower inflation, a significant build up of reserves, and a reduction of external debt are among the notable economic achievements. These developments benefited from favorable external conditions. Broad and ambitious structural reforms led to an improvement in the policy framework and institutions, leaving a strong legacy for the new administration that took office on May 29.

\section{The robust growth observed in recent years-supported by improved} macroeconomic conditions and structural reforms-is expected to continue in $2007 .{ }^{2}$ In 2006, solid agriculture and trade growth boosted non-oil sector activity. In 2007, supply-side conditions could improve as several new power plants should stabilize and enhance electricity production, and thus reduce production costs. Following a drop in crude oil production in 2006 due to unrest in the Niger Delta, a rebound is projected to the 2005 levels stemming mainly from production in new off-shore fields. Gas production will increase with the new West African Gas Pipeline.
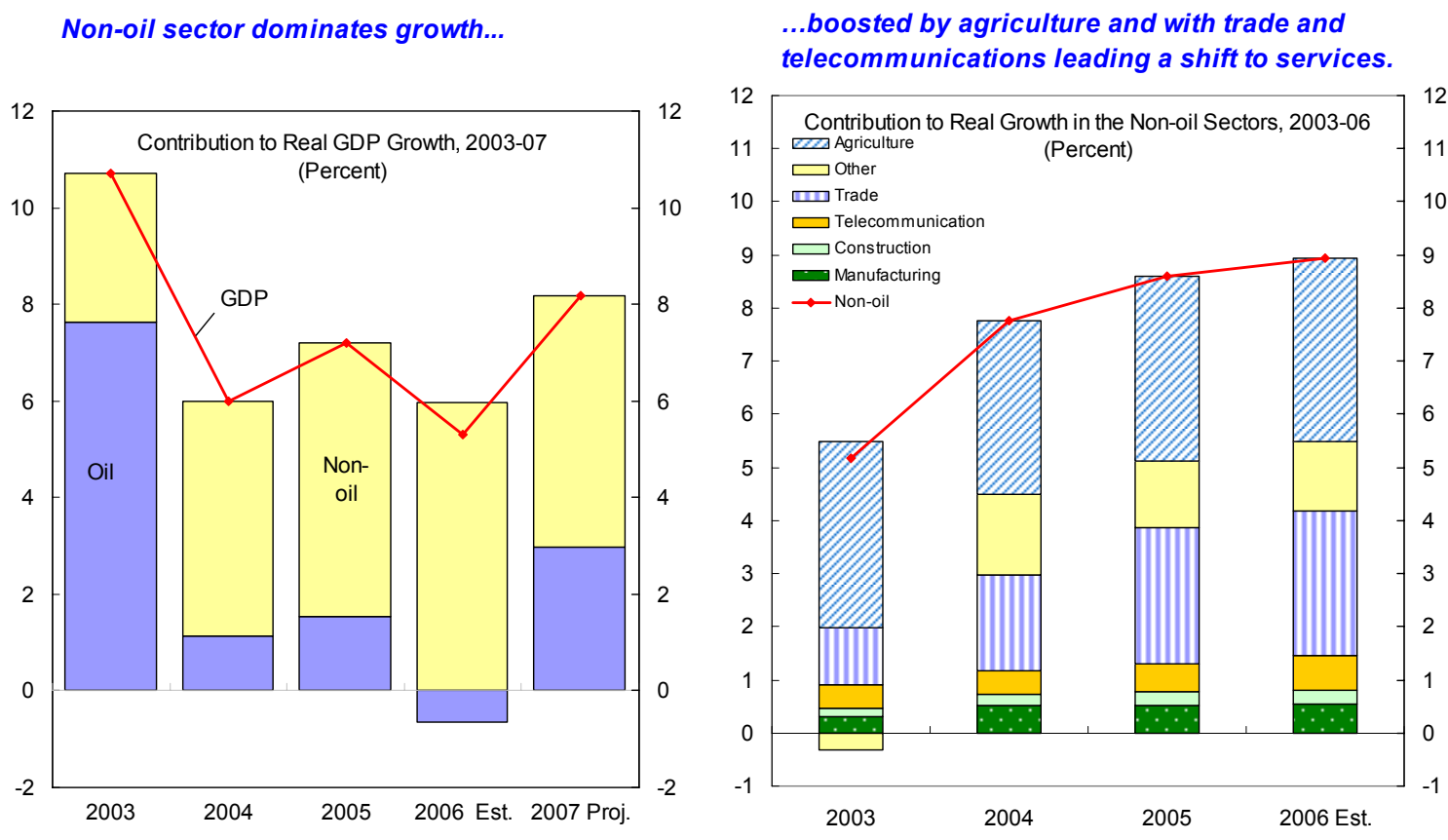

\footnotetext{
${ }^{1}$ The mission team comprised Mr. Nellor (Head), Ms. Murgasova, Ms. Roehler and Mr. Steinberg (all AFR), Mr. Williams (FAD), and Mr. Kelmanson (PDR). Mr. Bell (Senior Resident Representative) assisted the mission.

${ }^{2}$ The authorities have reestimated the GDP time series to integrate new data sources and methodological changes reflecting their efforts to implement SNA 1993. The staff plans to adopt a revised GDP time series at the next Article IV consultation. The revisions increase the level and share of non-oil GDP. Growth rates are broadly unchanged. Using the authorities' weights, real GDP growth would be 6.2 percent in 2006, and 8.4 percent in 2007. The authorities' policy statement (Appendix I) reports GDP according to the revised methodology.
} 
3. Following the Paris Club debt relief and aided by higher oil prices, the external position strengthened further through efforts to divest external debt and continued increases in international reserves. The London Club par bonds were repaid in late 2006, the liabilities for promissory notes were divested in March 2007, and efforts were made to buy back the oil warrants. Rising investor confidence is reflected in Nigeria's favorable sovereign ratings and interest rates on private sector external bond issuance.

High oil revenues and the Paris Club arrangement led to debt reduction and reserve accumulation...

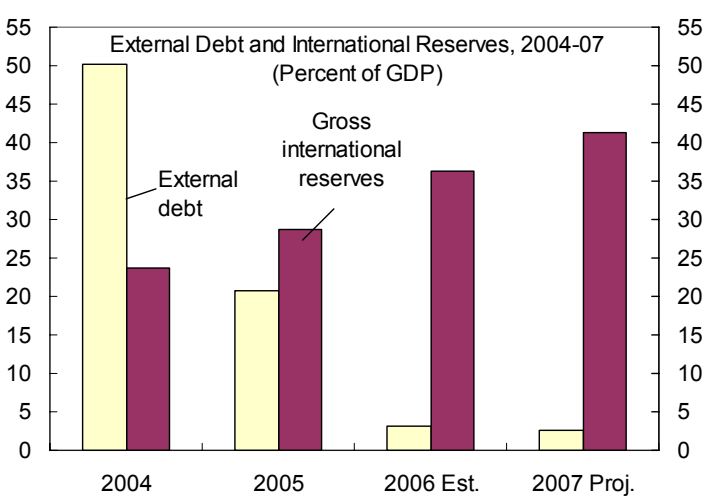

... and favorable international ratings of BB - .

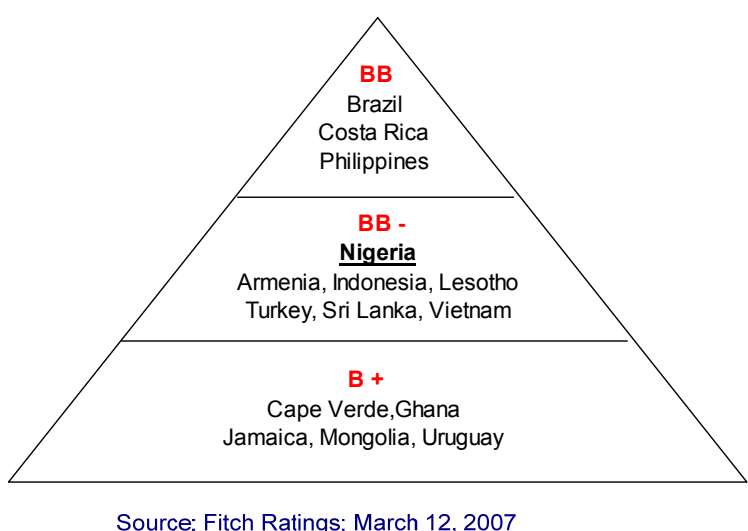

4. The immediate policy challenge is to manage the economy through the period of political transition: to preserve the gains of previous years, and provide a sound policy framework as the starting point for the incoming administration. Discussions with the authorities on the third review therefore centered on the following issues:

- $\quad$ Maintaining a macroeconomic policy setting consistent with stability;

- $\quad$ Safeguarding wealth through comprehensive asset and liability management; and

- $\quad$ Consolidating and institutionalizing reforms.

\section{Maintaining Macroeconomic Stability}

5. Against a background of the strong real and external position, fiscal and monetary policies were more expansionary than programmed in 2006. Growth of the monetary aggregates accelerated owing partly to ineffective monetary policy and a one-time increase in state spending. However, the unanticipated liquidity injection did not translate fully into inflation likely due to rising confidence and money demand. Inflation performance has improved including in relation to program projections. The 2007 macroeconomic program targets a stronger fiscal position and addresses the remaining risk of inflation by targeting monetary growth that is lower than non-oil sector growth. 


\section{A. Managing Fiscal Developments}

\section{The larger-than-projected expansion at the consolidated government level and the change in the composition of spending added to domestic demand in 2006.}

\section{- While the assessment criterion on the federal government fiscal balance was met with a margin, the target on the non-oil primary deficit of the consolidated government was exceeded by 3 percent of non-oil GDP (Tables 2a and 2c, Text Table 1). The expansion was due to higher-than-projected state and local government (SLG) spending financed by one-off distribution from the excess crude account beyond the oil price fiscal rule, a partial execution of the new explicit fuel subsidy scheme and somewhat higher gas receipts. ${ }^{3,4}$ Yet the overall fiscal balance remained well in surplus, enabling further accumulation of oil savings.}

Text Table 1. Consolidated Government (non-oil), 2005-07

(Percent of non-oil GDP)

\begin{tabular}{|c|c|c|c|c|c|}
\hline & \multirow{2}{*}{$\begin{array}{r}2005 \\
\text { Act. }\end{array}$} & \multicolumn{2}{|c|}{2006} & \multicolumn{2}{|c|}{2007} \\
\hline & & Prog. & Est. & Prog. & Proj. \\
\hline Non-oil revenue & 13.9 & 13.1 & 12.7 & 13.5 & 13.3 \\
\hline Non-oil expenditures & 59.5 & 60.7 & 62.9 & 59.1 & 60.0 \\
\hline Interest & 6.7 & 3.5 & 5.2 & 3.2 & 3.3 \\
\hline Federal government primary recurrent and extrabudgetary & 15.5 & 14.9 & 15.7 & 14.6 & 15.0 \\
\hline Federal government capital; and large projects & 6.3 & 12.6 & 10.5 & 11.7 & 12.1 \\
\hline of which : foreign content of large infrastructure projects & 0.6 & 3.2 & 2.1 & 3.1 & 3.2 \\
\hline State and local governments & 31.0 & 27.8 & 31.4 & 27.2 & 27.8 \\
\hline Explicit fuel subsidy & 0.0 & 2.1 & 0.1 & 2.3 & 1.8 \\
\hline Non-oil primary balance (excluding fuel subsidy) & -38.9 & -42.0 & -44.9 & -40.1 & -41.6 \\
\hline Proxy domestic balance $^{1}$ & -41.5 & -41.8 & -45.0 & -39.9 & -40.7 \\
\hline \multicolumn{6}{|l|}{ Memorandum items: } \\
\hline Overall balance (including oil revenue and expenditure) & 22.3 & 24.1 & 17.1 & 15.5 & 7.3 \\
\hline Balance excess crude account & 20.4 & $\ldots$ & 23.0 & $\ldots$ & $\ldots$ \\
\hline Non-oil GDP (billions of naira) & 6,223 & 7,305 & 7,310 & 8,549 & 8,446 \\
\hline
\end{tabular}

- $\quad$ The overall fiscal expansion combined with the underexecution of import-intensive capital spending raised the (proxy) domestic balance beyond projected levels. While identified capital spending increased relative to 2005 as intended, it remained below budget levels for the federal government and the large infrastructure projects.

\footnotetext{
${ }^{3}$ Since 2006, the oil price fiscal rule (see Country Report No. 07/20) has been interpreted as a revenue rule to compensate for both shortfalls from budgeted oil prices and production.

${ }^{4}$ The composition of state spending in 2006 will become available with a considerable lag.
} 
7. The authorities have reaffirmed their 2007 fiscal program set at the second review, which now involves a reduction of the fiscal impulse. The program envisages a reduction in the non-oil primary deficit of the consolidated government by about 3 percent of non-oil GDP. Capital spending needed to close Nigeria's infrastructure gap, including the large infrastructure projects with high import content, is expected to grow, while other spending remains contained. The assessment criterion for the federal government balance set at the second review has been reaffirmed by the authorities. Achievement of the consolidated government target hinges on adherence to the oil price fiscal rule by all levels of government; it

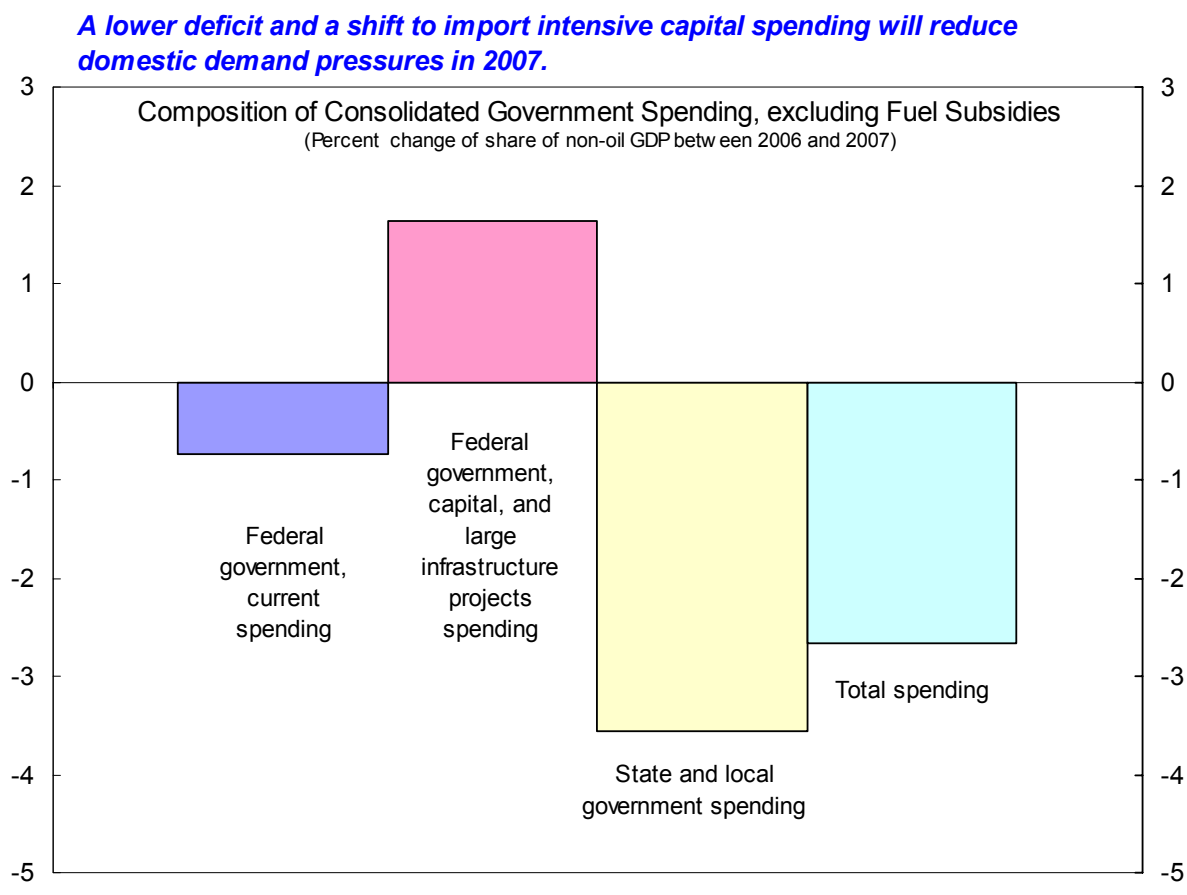
has been implemented so far in 2007.

\section{B. Strengthening Monetary Policy Implementation}

\section{Monetary targets were not met because policy only partially offset the unexpected} fiscal stimulus of late-2006.

- $\quad$ Problems with monetary data impeded effective analysis, thereby limiting the CBN's ability to respond promptly to changes in liquidity conditions (Box 1). The endDecember 2006 accounts have now been reconciled, including the previously-reported inconsistency on international reserves, and the external auditors gave an unqualified audit certification for the $2006 \mathrm{CBN}$ accounts. ${ }^{5}$

\section{Box 1. Monetary Statistics}

The simultaneous introduction of several new software systems at the CBN in January 2006 resulted in significant accounting errors, difficulties in preparing monetary statistics, and substantial delays in data reporting to the Fund throughout the year. The full extent and interrelationship of the problems became apparent during the external audit of the CBN's 2006 financial accounts. The Fund provided technical assistance both on accounting and statistics. The CBN has embarked on a comprehensive approach to resolve these issues related to accounting, statistics, and information technology (IT).

\footnotetext{
${ }^{5}$ Reconciliation of different sources of data on international reserves for early 2007 is ongoing.
} 
- Uneven implementation of monetary policy led to higher-than-programmed growth of monetary aggregates. With real interest rates remaining negative, reserve money was on average above program targets, and end-quarter corrections were only temporary. Insufficient sterilization of balance of payments inflows, reflecting higher than expected government spending, meant that the end-December reserve money target (assessment criterion) was exceeded (Text Table 2). In addition, reserve money was lowered through a reduction in the cash reserve requirement (CRR) - contrary to the CBN's Monetary Policy Committee communiqué in February 2006 - though the liquidity impact was sterilized with 91-day treasury bills. ${ }^{6}$ Adjusting for the reduction in the CRR, the reserve money target would have been surpassed by a wider margin. The authorities attribute the sharp rise in reserve money in December 2006 to a surge in currency resulting from demands associated with political campaigns. The surge in currency has been reversed in the first months of 2007 and hence represents a temporary deviation from the program.

- $\quad$ Allowing the naira to appreciate would have provided scope for greater foreign exchange sales to meet the reserve money target. The naira-U.S. dollar exchange rate has remained stable as a result of the authorities' intervention policies (Text Figure).

Text Table 2: Required Mop-up of Government Spending, 2004-07 ${ }^{1}$ (Percent of non-oil GDP)

\begin{tabular}{|c|c|c|c|c|c|c|c|c|c|c|}
\hline & \multirow{2}{*}{$\begin{array}{c}2004 \\
\text { Act. }\end{array}$} & \multirow{2}{*}{$\begin{array}{r}2005 \\
\text { Act. }\end{array}$} & \multicolumn{6}{|c|}{2006} & \multicolumn{2}{|c|}{2007} \\
\hline & & & Prog. & Q1 & Q2 & Q3 & Q4 & Act. & Prog. & Proj. \\
\hline Change in reserve money & 0.9 & 0.5 & 0.8 & -0.5 & 0.9 & 0.1 & 1.4 & 2.0 & 1.1 & 0.5 \\
\hline Proxy for domestic balance & -37.9 & -41.5 & -41.8 & -9.5 & -11.0 & -12.3 & -12.2 & -45.0 & -39.9 & -40.7 \\
\hline Mop-up & 37.1 & 41.0 & 41.0 & 9.9 & 10.1 & 12.2 & 10.8 & 43.0 & 38.8 & 40.2 \\
\hline Of which: foreign exchange sales & 25.8 & 21.5 & 16.5 & 1.4 & 4.9 & 6.4 & 7.8 & 20.5 & 20.0 & 26.3 \\
\hline domestic asset sales & $\ldots$ & $\ldots$ & $\ldots$ & 2.4 & 6.3 & 3.4 & -2.8 & 9.4 & $\ldots$ & $\ldots$ \\
\hline
\end{tabular}

Sources: Nigerian authorities, and Fund staff estimates and projections.

${ }^{1}$ Staff does not have adequate historical data on liquidity management to directly measure the scale of mop-up. This table uses the non-oil overall balance and the import content of large investment projects to proxy the domestic balance. The proxy for central bank intervention is, thus, the difference between the proxy domestic balance and the change in reserve money. It is indicative because it also captures the impact of the balance of payments, central bank profits, and other factors.

\footnotetext{
${ }^{6}$ The CRR was lowered from 5 to 0 percent on foreign currency deposits in October and from 5 to 3 percent on all other deposits in December.
} 

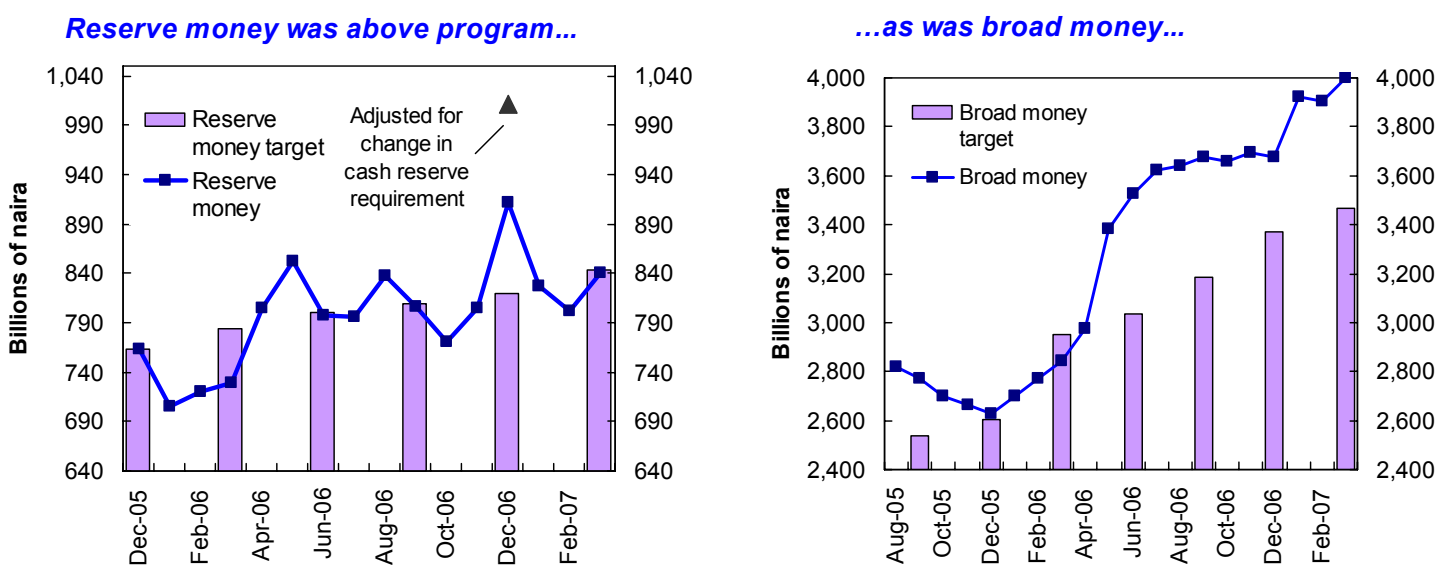

...resulting in looser monetary conditions, a stable exchange rate, and lower yields.

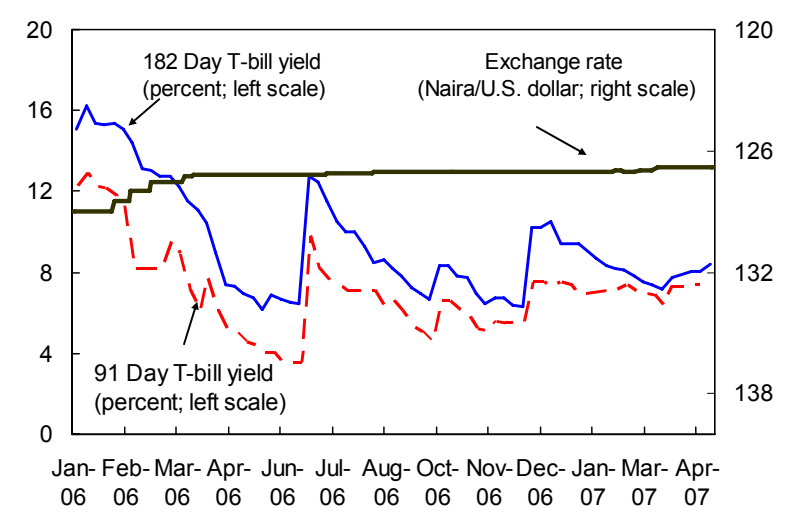

9. The inflation consequence of the growth in monetary aggregates was likely muted by an unexpected pick-up in money demand. Credit to the private sector, including long-term investment in the oil sector with limited domestic demand impact, explains about two-thirds of the broad money increase in mid-2006. Nevertheless, core inflation (excluding food and energy) accelerated somewhat in the last quarter of 2006. This increase was broad based, suggesting that it may have been related to the mid-2006 surge of liquidity. However, the subsequent decline in inflation in 2007 indicates that those inflationary pressures have diminished. This could be explained by a pick-up in money demand owing to a significant reduction in headline inflation and reemerging confidence in the financial system following the consolidation of the banking sector.

10. Against this background, the 2007 monetary program targets the CBN's single-digit inflation objective by reversing in part the acceleration in broad money. Broad money is targeted to grow by about 12 percent, which is below nominal non-oil GDP growth. Inflation developments will nonetheless need to be monitored closely. If money demand in 2007 turns out to be lower than projected or the impact of the monetary surge in mid-2006 is yet to be felt, monetary policy would have to be tightened to contain inflationary pressures. CBN's sterilization efforts should support the reserve money target by focusing on foreign exchange sales that would also allow sufficient domestic credit growth to the private sector and import growth consistent with the projected real appreciation. Greater flexibility in line with market conditions in the nominal exchange rate (compared to the current practice) and the nominal interest rates is essential. 
Confidence and money demand increased as average headline inflation fell.

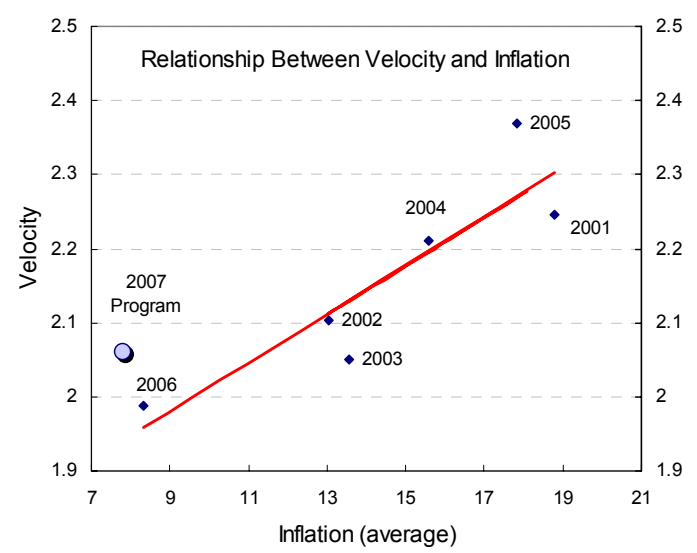

... as core inflation was broad based in late 2006, with 50 percent of core-urban commodities registering inflation in double digits.
This was largely driven by low food inflation...

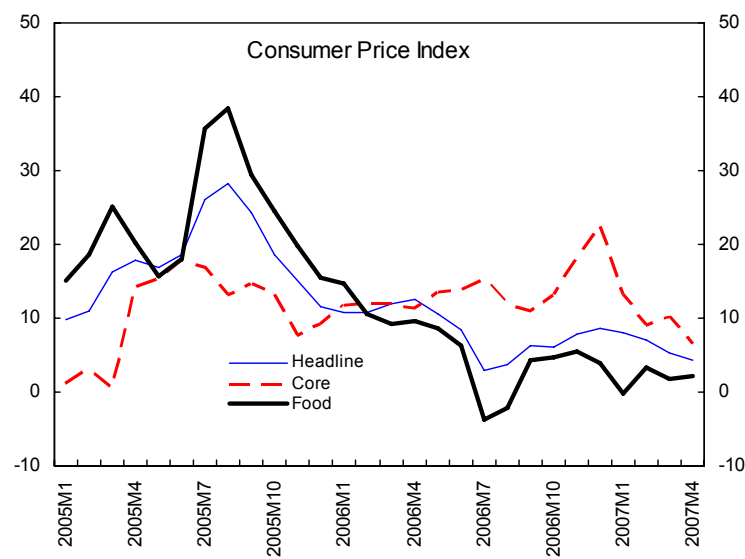

Sterilization through foreign exchange sales should encourage external adjustment - a faster real appreciation and further import growth.
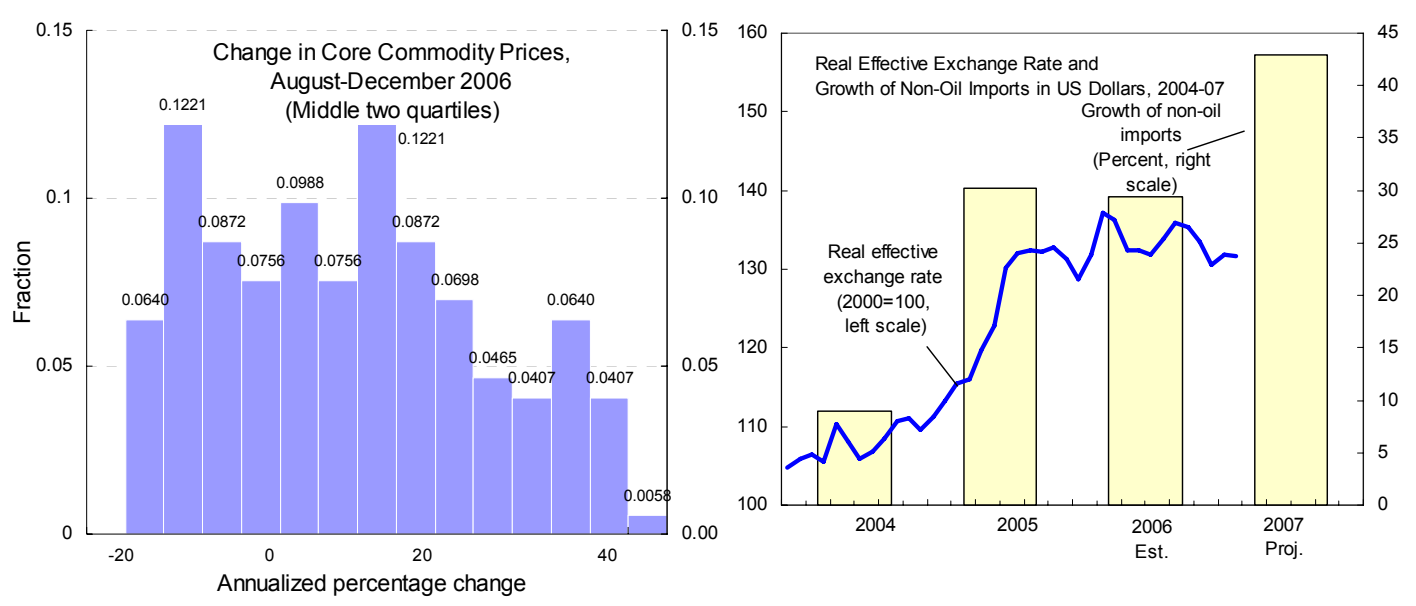

11. To improve the implementation of monetary policy, the CBN has introduced the following measures.

- $\quad$ Outstanding monetary data problems are being addressed. External advisors are assisting the CBN with IT issues and the Fund is providing technical assistance on accounting and monetary statistics. Regular data submissions of monthly data to the Fund have resumed.

- $\quad$ The monetary decision-making process is being strengthened, including through internal reorganization of the CBN. Liquidity management is being improved through effective operations of the fiscal and liquidity assessment groups, which should provide timely information on liquidity injections. The Fund's peripatetic expert and long-term advisor are assisting the process.

- Intra-month interest rate volatility should be reduced as a result of the new standing facility (end-June 2007 assessment criterion observed well ahead of schedule). 


\section{SAfeguarding Wealth Through Asset And Liability Management}

12. Nigeria significantly reinforced the management of its oil wealth in recent years. The oil price fiscal rule that limits the use of oil revenues and participation in the EITI were critical reforms for improving macroeconomic conditions and management of oil revenues. The approval of the Fiscal Responsibility and NEITI Bills by both houses of the parliament (and now awaiting harmonization) are welcome steps toward institutionalizing these reforms. In addition, setting the oil price fiscal rule consistent with macroeconomic stability, its possible expansion to natural gas, and plans to pursue fiscal responsibility bills for states are recognized by the outgoing administration as near-term priorities.

\section{A key challenge going forward is to develop and implement guidelines on the use} of Nigeria's oil savings. A recent stakeholder workshop on Oil Savings and the

Infrastructure Gap recommended clarifying the principles guiding the use of the oil savings as well as improving cost-benefit analysis and budget management to ensure high quality of spending, especially for joint federal and state large infrastructure projects (Box 2). These reforms will have to be advanced by the incoming administration.

\section{Box 2. Key Issues Raised by the Workshop on Oil Savings and the Infrastructure Gap ${ }^{1}$}

Improve the institutional framework for managing the oil savings

- $\quad$ Strengthen the oil price fiscal rule by defining the budget oil price in line with macroeconomic stability and extend it to natural gas.

- Define the objective of the oil savings "excess crude" account (stabilization and/or savings), governance arrangements, draw-down rules, and ensure transparent implementation.

- Develop asset management guidelines.

\section{Strengthen public financial management practices}

- Integrate all spending funded from oil savings into fiscal strategies, budgets, and accounts.

- Make cost-benefit analysis an integral component of the project selection process.

- Establish participatory arrangements for the management of joint national and state projects and ensure application of due process standards.

${ }^{1}$ Workshop documents are published on http://www.fmf.gov.ng.

\section{A comprehensive debt management framework is being prepared to preserve} recent gains in debt sustainability. It will provide guidance to policy makers and agencies on the government's borrowing strategy, procedures, and acceptable terms and conditions of loans. The new government will need to ensure effective implementation of the framework, including at subnational levels. ${ }^{7}$

\footnotetext{
${ }^{7}$ A Memorandum of Understanding (MOU) with China signed in 2006 on a framework for negotiations of infrastructure loans, is being passed to the new administration for its consideration. The current government views the MOU as in its national interest including as an important element of addressing the infrastructure gap.
} 


\section{Consolidating Structural Reforms}

\section{Under the PSI, the authorities successfully implemented their ambitious}

structural reforms (Text Table 3). The reforms, based on the National Economic Empowerment and Development Strategy (NEEDS) focused on increasing the efficiency of the public sector and creating an enabling environment for the private sector. Good progress has been made in reorganizing the government and parastatals; rightsizing the civil service and improving its skill mix; strengthening public financial management, privatizing public enterprises, and removing obstacles for private sector led growth. To ensure policy continuity, government ministries have prepared policy strategy documents for the incoming administration.

Text Table 3. Main Accomplishments of the Structural Reform Program under the PSI, 2005-07 ${ }^{1}$

\begin{tabular}{|c|c|}
\hline \multicolumn{2}{|c|}{ Improve macroeconomic management } \\
\hline Fiscal policy & $\begin{array}{l}\text { Oil price fiscal rule applied. } \\
\text { Fiscal Responsibility Bill passed by both houses of parliament, awaiting harmonization. }\end{array}$ \\
\hline Foreign exchange markets & $\begin{array}{l}\text { Whole Sale Dutch Auction System operational. } \\
\text { Exchange markets unified. }\end{array}$ \\
\hline Monetary policy & Standing facility introduced. \\
\hline \multicolumn{2}{|c|}{ Public financial management reforms to support macroeconomic and fiscal management } \\
\hline Multi-year, strategic budgeting & $\begin{array}{l}\text { Federal and state government strategies (NEEDS and SEEDS) guide policies. } \\
\text { NEEDS review and SEEDS benchmarking carried out. } \\
\text { Medium-term sector strategies expanded. }\end{array}$ \\
\hline Budget accountability & $\begin{array}{l}\text { Virtual poverty fund for debt relief-financed MDG spending introduced. } \\
\text { Virtual poverty fund quarterly reports initiated. } \\
\text { All contractor and almost all pension arrears cleared. }\end{array}$ \\
\hline Computerized systems & $\begin{array}{l}\text { Electronic accounting system (ATRRS) introduced to government agencies. } \\
\text { Integrated Personnel and Payroll System for federal government rolled out to several agencies. } \\
\text { Human resources management projects in tax and customs administration underway. }\end{array}$ \\
\hline \multicolumn{2}{|c|}{ Reform of tax and customs administration to ensure revenue collection while being business friendly } \\
\hline Tax administration & $\begin{array}{l}\text { Internal reorganization, and greater independence established. } \\
\text { New taxpayer database and taxpayer enumeration project underway. }\end{array}$ \\
\hline Customs service & Fast-track customs clearance procedures for low risk clients expanded. \\
\hline \multicolumn{2}{|c|}{ Governance, transparency, and the effectiveness of government operations } \\
\hline Oil revenue & $\begin{array}{l}\text { Oil revenue reports and reconciliation beyond EITI requirements published. } \\
\text { NEITI bill passed by both houses of parliament, awaiting harmonization. }\end{array}$ \\
\hline Procurement reform & $\begin{array}{l}\text { "Due process" procedures including central monitoring of tendering and pre-payments certification enforced. } \\
\text { Procurement bill passed by both houses of parliament, awaiting harmonization. } \\
\text { Procurement manual prepared. }\end{array}$ \\
\hline Corruption & Economic and Financial Crimes Commission (EFCC) active including cases against many state governors. \\
\hline Civil service & $\begin{array}{l}\text { Large scale disengagement of staff without relevant skills, and selective hiring of high skilled staff. } \\
\text { Wage reform consolidated most allowances, and provides incentives only for selected scarce skills. }\end{array}$ \\
\hline Government administration & Internal reorganization and streamlining of all government agencies and many parastatals advanced. \\
\hline \multicolumn{2}{|c|}{ Redefine the role of government in the economy in support of private sector-led growth } \\
\hline Business environment, trade & $\begin{array}{l}\text { Tariff reform reduced unweighted average tariff below } 20 \text { percent. } \\
\text { All major ports concessioned. } \\
\text { Investor one-stop shop set up. } \\
\text { Domestic petroleum product markets partly liberalized through adjustment to domestic fuel prices, and an explicit } \\
\text { fuel subsidy scheme. }\end{array}$ \\
\hline Privatization & $\begin{array}{l}\text { Power sector reorganized, and evaluation of bidders for many power sector companies underway. } \\
\text { Large petrochemical and most oil service companies privatized. Refineries and other oil companies underway. } \\
\text { Fixed line telephone company privatized. } \\
\text { Hotels and other commercial enterprises privatized. }\end{array}$ \\
\hline \multicolumn{2}{|c|}{ Strengthen the financial system } \\
\hline Banking sector & Bank consolidation (89 to 25 banks) and recapitalization completed. \\
\hline
\end{tabular}


16. The final steps in completing several structural measures were delayed in the last few months, despite the good progress in implementation of most cases (Table 8). One structural assessment criterion for the third review was observed on time, while five criteria were delayed (Text Table 4). Of the structural benchmarks for December 2006 and March 2007 four were observed, one was observed with a delay, and two were delayed. Most of the delayed measures are well advanced and the authorities are pressing ahead to complete them on a revised timetable. At the same time, one assessment criterion and two structural benchmarks for June 2007 have already been completed.

Text Table 4. Nigeria: Structural Assessment Criteria for the 3rd Review

\begin{tabular}{|c|c|}
\hline $\begin{array}{l}\text { Structural Assessment Criterion } \\
\text { (Target Date) }\end{array}$ & Comments \\
\hline $\begin{array}{l}\text { Establish database of pensioners and } \\
\text { estimate size of pension arrears } \\
\text { (September 2006). }\end{array}$ & Observed. \\
\hline $\begin{array}{l}\text { Introduce Integrated Personnel and Payroll } \\
\text { Information System (IPPIS) (September } \\
\text { 2006). }\end{array}$ & $\begin{array}{l}\text { Not met. Waiver requested. While delayed, now substantively } \\
\text { implemented. Software installation in the } 6 \text { ministries and } 6 \text { central } \\
\text { agencies of the } 1 \text { st phase completed in March. Test payroll processing } \\
\text { with the new system commenced in April, and deployment expected } \\
\text { soon. }\end{array}$ \\
\hline $\begin{array}{l}\text { The Central Bank of Nigeria to establish } \\
\text { prudential standards for consolidated } \\
\text { supervision and begin to supervise the } \\
\text { banking groups on a consolidated basis } \\
\text { (December 2006). }\end{array}$ & $\begin{array}{l}\text { Not met. Waiver requested. Remedial measures include a time-bound } \\
\text { action plan by the time of the fourth review, and plans for a long-term } \\
\text { technical assistance expert from mid-2007. Unanticipated problems } \\
\text { related to post-consolidation of banks emerged and precluded the } \\
\text { immediate implementation of consolidated supervision. }\end{array}$ \\
\hline $\begin{array}{l}\text { FIRS to conduct nationwide taxpayer } \\
\text { enumeration in preparation for introducing } \\
\text { automated tax administration system, } \\
\text { including TIN (December 2006). }\end{array}$ & $\begin{array}{l}\text { Not met. Waiver requested as scale of necessary work was expanded } \\
\text { and expected observed by July } 2007 \text {. Delay due to an unanticipated } \\
\text { second exercise to verify basic taxpayer information. }\end{array}$ \\
\hline $\begin{array}{l}\text { Appoint auditors to conduct the audit of } \\
\text { the oil and gas sector for } 2005 \text { and } 2006 \\
\text { (March 2007). }\end{array}$ & $\begin{array}{l}\text { Not met. Waiver requested. Observed for } 2005 \text { audit. Delay with } \\
\text { contract unlikely to hold up the } 2006 \text { audit. For the } 2006 \text { audit, open } \\
\text { procurement has been launched. }\end{array}$ \\
\hline $\begin{array}{l}\text { Management contract for the } \\
\text { Transmission company of Nigeria will be } \\
\text { awarded (March 2007). }\end{array}$ & $\begin{array}{l}\text { Not met. Waiver requested as delay is short. Expected observed by } \\
\text { end-May. Delays were caused by procedure requirements in this World } \\
\text { Bank sponsored project. Expressions of interest were evaluated, and } \\
\text { bid documents issued to four pre-qualified companies. }\end{array}$ \\
\hline
\end{tabular}

Sources: Nigerian authorities, and Fund staff assessment.

17. The substantive completion of a major banking sector consolidation allows the CBN to move forward with measures to enhance financial stability and development. Next steps include strengthening banking supervision and carrying out a financial stability diagnostic. Fund technical assistance will support supervision of banking groups on a consolidated basis. The $\mathrm{CBN}$ is also developing a medium-to-long-term financial sector strategy (FSS 2020) with Fund and Bank input.

18. NEEDS 2 for 2008-11 - under preparation-will provide a draft policy strategy for the new administration. NEEDS 2 will be based on wide-ranging consultations, and a review of the current NEEDS (see progress report and JSAN (forthcoming)). It can also draw 
on the benchmarking exercise of the state-level strategies (the SEEDS), the federal government Medium Term Sector Strategies (MTSS) prepared for the 2007 budget, and the performance reports on MDG-related spending financed from Paris Club debt relief.

\section{PSI IsSUES}

19. To monitor Nigeria's performance under the PSI, the following quantitative and structural assessment criteria and quantitative benchmarks are being proposed through end-June 2007.

- $\quad$ The assessment criterion on the non-oil primary balance of the federal government set at the second review is reconfirmed. While significant spending slippages took place in the SLGs, broadening the coverage of the assessment criterion in not envisaged because the federal government has no control over SLG spending.

- $\quad$ The reserve money and net foreign assets targets are revised in light of new information for 2006 and new oil price projections.

- $\quad$ All structural assessment criteria and structural benchmarks set at the second review remain valid.

20. The staff recommends the following waivers of nonobservance in order to complete the third PSI review. Waivers are recommended for the nonobservance of five structural assessment criteria and two quantitative assessment criteria on reserve money and nonconcessional external borrowing (Text Table 4). Regarding the latter, a nonconcessional loan of \$200 million was contracted in January 2006 to finance a communication satellite. This loan (0.2 percent of GDP) represents a small deviation from the program and does not substantially weaken Nigeria's debt sustainability. The supplier's contract was signed in 2004 and the satellite was launched in May 2007.

\section{Staff Appraisal}

\section{The Nigerian authorities' reform program has led to a robust macroeconomic} performance in recent years. The improved policy framework, strengthened institutions, stronger macroeconomic policies, and major progress with wide-ranging structural reforms have resulted in impressive macroeconomic outcomes - notably strong growth and low inflation - as well as improved investor confidence. These results leave a strong legacy for the incoming government.

22. The more recent slippages in policy implementation are addressed adequately in the 2007 program, thereby minimizing the risks to macroeconomic performance. The fiscal expansion at the consolidated government level in late 2006 resulted from a regrettable deviation from the oil price fiscal rule. In conjunction with the lack of exchange rate flexibility, in contrast to previous commitments, this led to an unanticipated liquidity 
injection. Nevertheless, this did not result in excessive inflationary pressures, due largely to increased confidence and likely higher money demand. The 2007 monetary program therefore targets single-digit inflation by partly reversing the broad money acceleration. The inflation objective should be supported by the overall fiscal contraction (which hinges on adherence to the oil price fiscal rule). To support this, staff urges the new authorities to commit to and pursue a more flexible exchange rate policy going forward. Inflation will need to be monitored closely and monetary conditions tightened in the event that money demand is not as strong as anticipated or the impact of the monetary surge in mid-2006 is yet to be felt. A number of measures being implemented by the CBN should help improve the effectiveness of monetary policy and preserve the low inflation environment. Significant data problems have persisted. Ensuring good quality of monetary data will be therefore essential. Similarly, the recent delays in completing the final steps of several structural measures, while regrettable, should not distract from the progress achieved in structural areas under the PSI.

\section{The main challenge for the new administration will be to maintain the reform} momentum and safeguard economic gains of recent years. Efforts should focus on three main areas. First, fiscal policy needs to remain consistent with macroeconomic stability. Reaffirming the political agreement on the use of oil revenues among all levels of government will be important in this regard. Political pressures, including from the states, to spend oil savings are likely to remain considerable. Second, recent efforts to develop and implement the guidelines on the effective use of oil savings and ensure good quality of capital spending need to be continued. Third, structural reforms need to be taken forward. Failure to pursue these reforms could jeopardize the macroeconomic stability and future growth potential, which are important elements in Nigeria's fight against poverty.

24. The staff recommends completion of the third review under the PSI (with the waivers and modifications described in section $\mathbf{V}$ ). The completion of this review will support Nigeria's efforts to entrench macroeconomic stability and pursue ongoing strong structural reforms. 
Table 1. Nigeria: Selected Economic and Financial Indicators, 2004-09

\begin{tabular}{|c|c|c|c|c|c|c|c|c|}
\hline & \multirow{2}{*}{$\begin{array}{r}2004 \\
\text { Act. }\end{array}$} & \multirow{2}{*}{$\begin{array}{r}2005 \\
\text { Act. }\end{array}$} & \multicolumn{2}{|c|}{2006} & \multicolumn{2}{|c|}{2007} & \multirow{2}{*}{$\begin{array}{c}2008 \\
\text { Proj. }\end{array}$} & \multirow{2}{*}{$\begin{array}{l}2009 \\
\text { Proj. }\end{array}$} \\
\hline & & & Prog. & Est. & Prog. & Proj. & & \\
\hline \multicolumn{9}{|c|}{ National income and prices (annual percentage change, unless otherwise specified) } \\
\hline Real GDP (at 1990 factor cost) & 6.1 & 7.2 & 5.1 & 5.2 & 7.3 & 8.2 & 6.7 & 5.7 \\
\hline Oil GDP & 3.5 & 4.2 & -1.6 & -2.8 & 9.3 & 9.9 & 7.1 & 3.7 \\
\hline Non-oil GDP & 7.4 & 8.6 & 8.2 & 8.9 & 6.5 & 7.5 & 6.5 & 6.6 \\
\hline Production of crude oil (million barrels per day) & 2.50 & 2.51 & 2.35 & 2.36 & 2.53 & 2.53 & 2.66 & 2.71 \\
\hline Nominal GDP at market prices (billions of naira) & 9,604 & 12,939 & 14,824 & 14,693 & 16,589 & 16,124 & 18,528 & 20,286 \\
\hline Nominal non-oil GDP at factor cost (billions of naira) & 5,005 & 6,223 & 7,305 & 7,310 & 8,549 & 8,446 & 9,781 & 11,262 \\
\hline Nominal GDP per capita (U.S. dollars) & 501 & 674 & 773 & 770 & 850 & 827 & 930 & 1,001 \\
\hline GDP deflator & 20.3 & 25.7 & 9.1 & 7.8 & 4.3 & 1.5 & 7.7 & 3.5 \\
\hline Non-oil GDP deflator & 14.5 & 14.5 & 8.5 & 7.8 & 9.9 & 7.5 & 8.7 & 8.1 \\
\hline Consumer price index (annual average) & 15.0 & 17.9 & 9.0 & 8.2 & 10.3 & 7.8 & 9.2 & 8.5 \\
\hline Consumer price index (end of period) & 10.0 & 11.6 & 12.2 & 8.5 & 9.5 & 9.9 & 9.0 & 8.0 \\
\hline \multicolumn{9}{|l|}{ Investment and savings (percent of GDP) } \\
\hline Gross national savings & 27.8 & 30.5 & 34.2 & 33.8 & 35.2 & 33.0 & 31.1 & 29.8 \\
\hline Public & 20.8 & 22.0 & 25.0 & 21.6 & 23.5 & 19.9 & 22.1 & 19.4 \\
\hline Private & 7.0 & 8.5 & 9.3 & 12.1 & 11.7 & 13.1 & 9.0 & 10.4 \\
\hline Investment & 22.5 & 21.2 & 21.8 & 22.0 & 24.8 & 25.9 & 23.7 & 23.7 \\
\hline Public & 9.1 & 9.6 & 10.4 & 10.3 & 12.7 & 13.4 & 11.4 & 11.2 \\
\hline Private & 13.4 & 11.6 & 11.4 & 11.7 & 12.1 & 12.5 & 12.4 & 12.6 \\
\hline Current account balance & 5.3 & 9.2 & 12.5 & 11.8 & 10.4 & 7.0 & 7.4 & 6.0 \\
\hline Public & 11.7 & 12.4 & 14.6 & 11.3 & 10.8 & 6.4 & 10.7 & 8.2 \\
\hline Private & -6.4 & -3.2 & -2.2 & 0.5 & -0.3 & 0.6 & -3.4 & -2.2 \\
\hline \multicolumn{9}{|c|}{ Consolidated government operations (consists of federal, state, and local governments; percent of GDP) } \\
\hline Total revenues and grants & 43.0 & 43.4 & 45.4 & 43.7 & 46.2 & 43.7 & 42.6 & 41.3 \\
\hline Of which: oil and gas revenue & 34.9 & 36.8 & 38.9 & 37.1 & 39.2 & 36.8 & 35.5 & 33.6 \\
\hline Total expenditure and net lending (commitment basis) & 35.3 & 34.2 & 33.4 & 34.9 & 38.2 & 39.9 & 34.0 & 35.1 \\
\hline Overall balance (commitment basis) & 7.7 & 9.3 & 12.0 & 8.8 & 8.0 & 3.8 & 8.6 & 6.2 \\
\hline Non-oil primary balance (percent of non-oil GDP) & -34.2 & -38.9 & -42.0 & -44.9 & -40.0 & -41.6 & -40.5 & -40.4 \\
\hline \multicolumn{9}{|c|}{ Money and credit (change in percent of broad money at the beginning of the period) } \\
\hline Broad money & 14.0 & 16.0 & 28.4 & 39.9 & 11.8 & 11.8 & 15.8 & .. \\
\hline Net foreign assets & 62.3 & 61.8 & 62.7 & 68.6 & 60.1 & 36.3 & 58.7 & $\ldots$ \\
\hline Net domestic assets & -49.9 & -45.8 & -34.3 & -28.7 & -48.3 & -24.5 & -42.9 & ... \\
\hline Credit to consolidated government & -42.1 & -17.4 & -35.1 & -34.5 & -42.6 & -20.9 & -40.7 & $\ldots$ \\
\hline Credit to the rest of the economy & 15.7 & 19.5 & 8.4 & 20.0 & 11.4 & 12.8 & 11.3 & $\ldots$ \\
\hline Velocity & 2.2 & 2.4 & 2.2 & 2.0 & 2.3 & 2.1 & 2.1 & $\ldots$ \\
\hline Treasury bill rate (percent; end of period) & 14.3 & 12.2 & $\ldots$ & 7.4 & $\ldots$ & $\cdots$ & $\cdots$ & $\cdots$ \\
\hline \multicolumn{9}{|c|}{ External sector (annual percentage change, unless otherwise specified) } \\
\hline Exports, f.o.b. & 36.8 & 42.6 & 11.3 & 15.9 & 8.5 & 3.3 & 10.5 & 2.5 \\
\hline Oil export volume & 2.5 & -3.5 & -7.0 & -2.7 & 8.7 & 6.8 & 2.0 & 1.8 \\
\hline Imports, f.o.b. & 13.1 & 30.5 & 19.5 & 21.5 & 20.6 & 26.5 & 8.1 & 7.3 \\
\hline Terms of trade & 20.5 & 38.0 & 16.5 & 16.4 & -2.3 & -5.6 & 5.4 & -1.5 \\
\hline Price of Nigerian oil (U.S. dollars per barrel) & 38.3 & 55.3 & 64.4 & 64.3 & 63.3 & 60.8 & 64.8 & 64.5 \\
\hline Nominal effective exchange rate (end of period) & -2.8 & 10.4 & $\ldots$ & -6.0 & $\ldots$ & $\cdots$ & $\cdots$ & $\cdots$ \\
\hline Real effective exchange rate (end of period) & 4.0 & 19.7 & $\ldots$ & -0.5 & $\ldots$ & $\ldots$ & $\ldots$ & $\ldots$ \\
\hline External debt outstanding (billions of U.S. dollars) & 35.9 & 20.5 & 3.5 & 3.5 & 3.7 & 3.3 & 3.9 & 4.7 \\
\hline Gross international reserves (billions of U.S. dollars) & 17.0 & 28.3 & 46.5 & 41.8 & 62.4 & 52.3 & 71.6 & 89.9 \\
\hline (equivalent months of imports of goods and services) & 6.0 & 8.5 & 12.0 & 10.2 & 14.2 & 11.9 & 15.3 & 18.3 \\
\hline
\end{tabular}

Sources: Nigerian authorities, and Fund staff estimates and projections. 
Table 2a. Nigeria: Consolidated Government (Cash Basis), 2004-09

(Billions of naira)

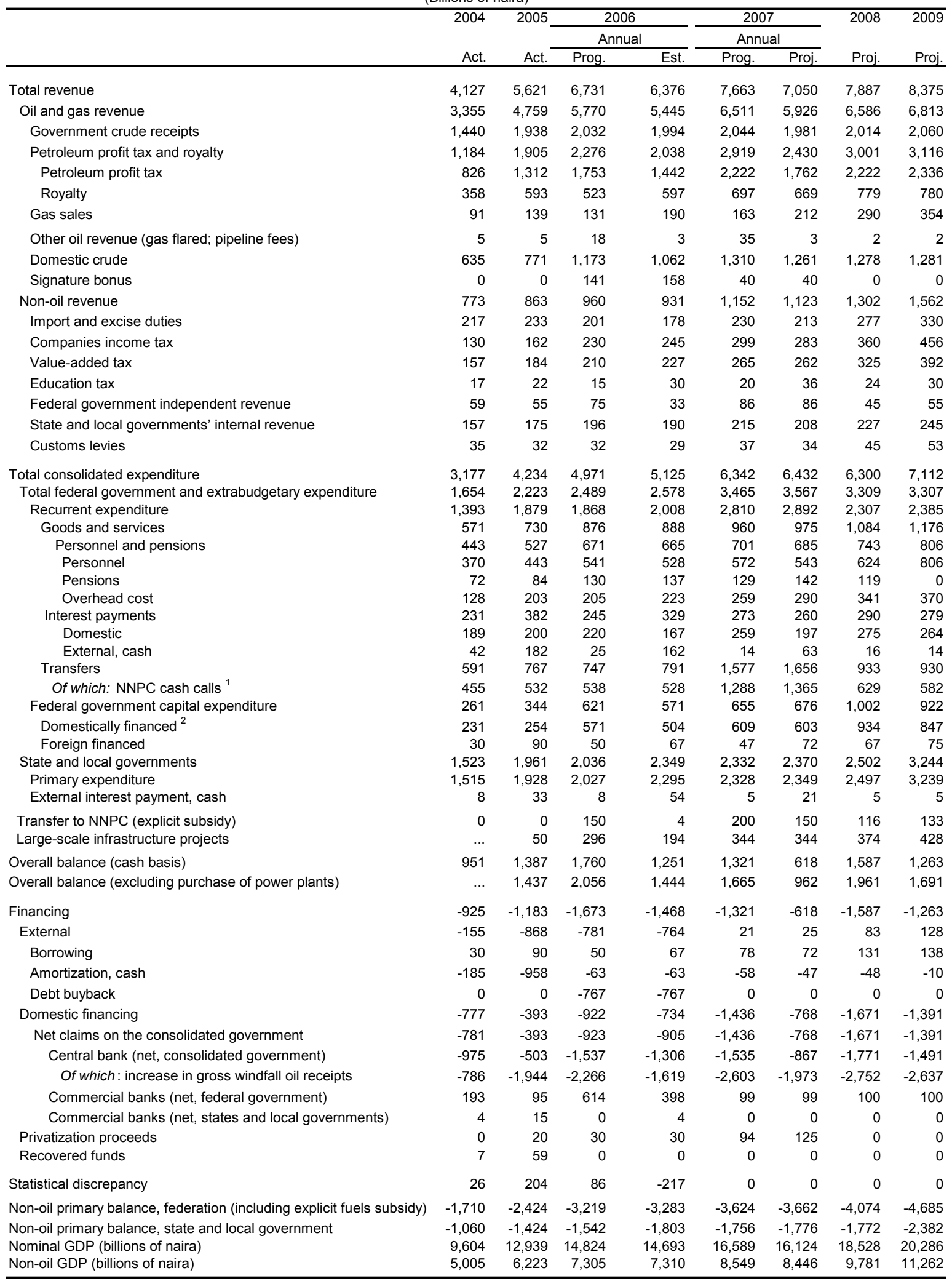

Sources: Nigerian authorities, and Fund staff estimates and projections

${ }^{1}$ Includes capital contributions to joint venture projects with oil companies for power plants.

${ }^{2}$ Actual cash spending 
Table 2b. Nigeria: Federal Government Budget, 2005-07

(Billions of naira)

\begin{tabular}{|c|c|c|c|c|c|}
\hline & \multirow{2}{*}{$\begin{array}{r}2005 \\
\text { Act. }\end{array}$} & \multicolumn{2}{|c|}{2006} & \multicolumn{2}{|c|}{2007} \\
\hline & & Prog. & Est. & Prog. & Proj. \\
\hline Total revenue $^{1}$ & 2,058 & 2,588 & 2,424 & 2,895 & 2,633 \\
\hline Petroleum revenue & 1,794 & 2,290 & 2,166 & 2,529 & 2,283 \\
\hline Nonpetroleum revenue & 264 & 298 & 258 & 366 & 350 \\
\hline Import and excise duties & 108 & 91 & 80 & 104 & 96 \\
\hline Companies' income tax & 76 & 104 & 114 & 139 & 132 \\
\hline Value-added tax & 25 & 28 & 31 & 37 & 36 \\
\hline Federal government independent revenue & 55 & 75 & 33 & 86 & 86 \\
\hline Total expenditure & 1,445 & 1,859 & 1,815 & 2,044 & 2,016 \\
\hline Recurrent expenditure & 1,192 & 1,288 & 1,311 & 1,435 & 1,413 \\
\hline Goods and services & 730 & 876 & 888 & 960 & 975 \\
\hline Personnel and pension & 527 & 671 & 665 & 701 & 685 \\
\hline Personnel & 443 & 541 & 528 & 572 & 543 \\
\hline Pensions & 84 & 130 & 137 & 129 & 142 \\
\hline Overhead cost & 203 & 205 & 223 & 259 & 290 \\
\hline Interest payments & 382 & 245 & 329 & 273 & 260 \\
\hline Domestic & 200 & 220 & 167 & 259 & 197 \\
\hline External, cash & 182 & 25 & 162 & 14 & 63 \\
\hline Transfers $^{2}$ & 79 & 167 & 94 & 202 & 177 \\
\hline Nigerian National Petroleum Corporation (NNPC) & 0 & 75 & 4 & 100 & 75 \\
\hline National Judicial Council & 33 & 35 & 35 & 43 & 43 \\
\hline Transfer to Niger Delta Development Commission & 22 & 26 & 26 & 24 & 24 \\
\hline Universal Basic Education Commission & 24 & 31 & 30 & 35 & 35 \\
\hline Capital expenditure & 254 & 571 & 504 & 609 & 603 \\
\hline Domestic & 254 & 571 & 504 & 609 & 603 \\
\hline Overall balance (cash basis) & 612 & 729 & 609 & 851 & 617 \\
\hline Overall balance (excluding purchase of power plants) & 635 & 729 & 609 & 851 & 617 \\
\hline Financing & -963 & $-1,008$ & $-1,080$ & -851 & -617 \\
\hline External & -716 & -486 & -485 & -43 & -35 \\
\hline Borrowing & 0 & 0 & 0 & 0 & 0 \\
\hline Amortization, cash & -716 & -48 & -47 & -43 & -35 \\
\hline Debt buyback & 0 & -438 & -438 & 0 & 0 \\
\hline Domestic financing & -326 & -552 & -624 & -902 & -707 \\
\hline Net claims on the federal government & -326 & -552 & -841 & 0 & 0 \\
\hline Central bank (net) & -421 & $-1,166$ & $-1,239$ & $-1,001$ & -806 \\
\hline Of which: gross oil windfall proceeds & -820 & -956 & -683 & $-1,098$ & -832 \\
\hline Commercial banks (net) & 95 & 614 & 398 & 99 & 99 \\
\hline Nonbank financing & 0 & 0 & 0 & 0 & 0 \\
\hline Privatization proceeds & 20 & 30 & 30 & 94 & 125 \\
\hline Of which: sales of real estate assets & 15 & 15 & 15 & 15 & 15 \\
\hline Recovered funds & 59 & 0 & 0 & 0 & 0 \\
\hline Statistical discrepancy & -350 & -280 & -471 & 0 & 0 \\
\hline \multicolumn{6}{|l|}{ Memorandum items: } \\
\hline Primary spending & 1,063 & 1,614 & 1,486 & 1,771 & 1,756 \\
\hline Non-oil primary balance (including explicit fuels subsidy) & -799 & $-1,316$ & $-1,228$ & $-1,405$ & $-1,405$ \\
\hline Non-oil primary balance (excluding purchase of power plants) & -799 & $-1,316$ & $-1,228$ & $-1,405$ & $-1,405$ \\
\hline Budgetary revenue & 2,058 & 2,588 & 2,424 & 2,895 & 2,633 \\
\hline Budgetary spending (including explicit fuels subsidy) & 1,396 & 1,907 & 1,862 & 2,087 & 2,052 \\
\hline Balance on the budget (BOF definition, debt service above the line) & 661 & 681 & 562 & 808 & 582 \\
\hline
\end{tabular}

Sources: Nigerian authorities, and Fund staff estimates and projections.

${ }^{1}$ Oil revenue net of cash call payments.

${ }^{2}$ Excluding transfer to the NNPC for cash call payments. Includes fuel subsidy payments to independent marketers. 


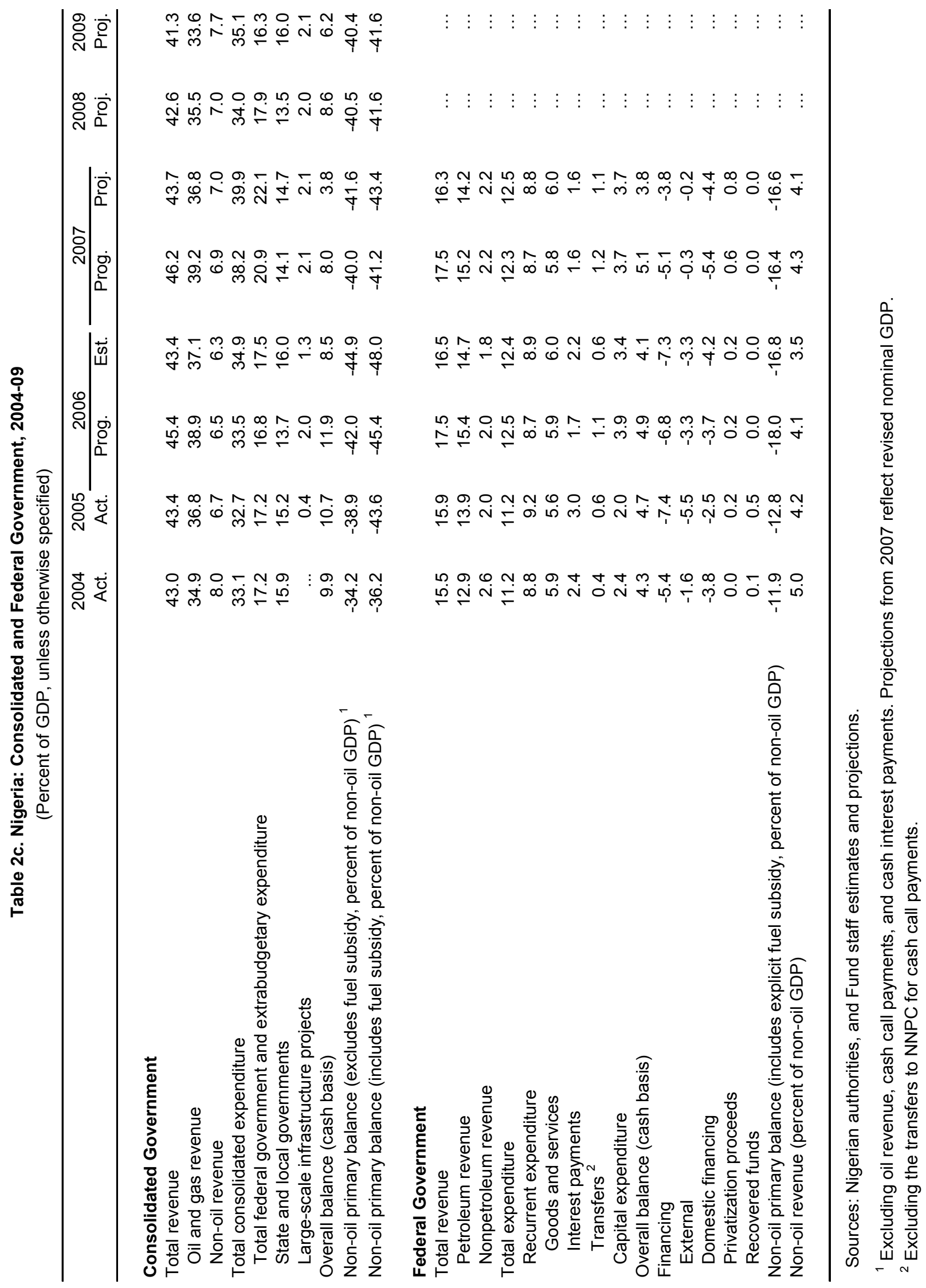




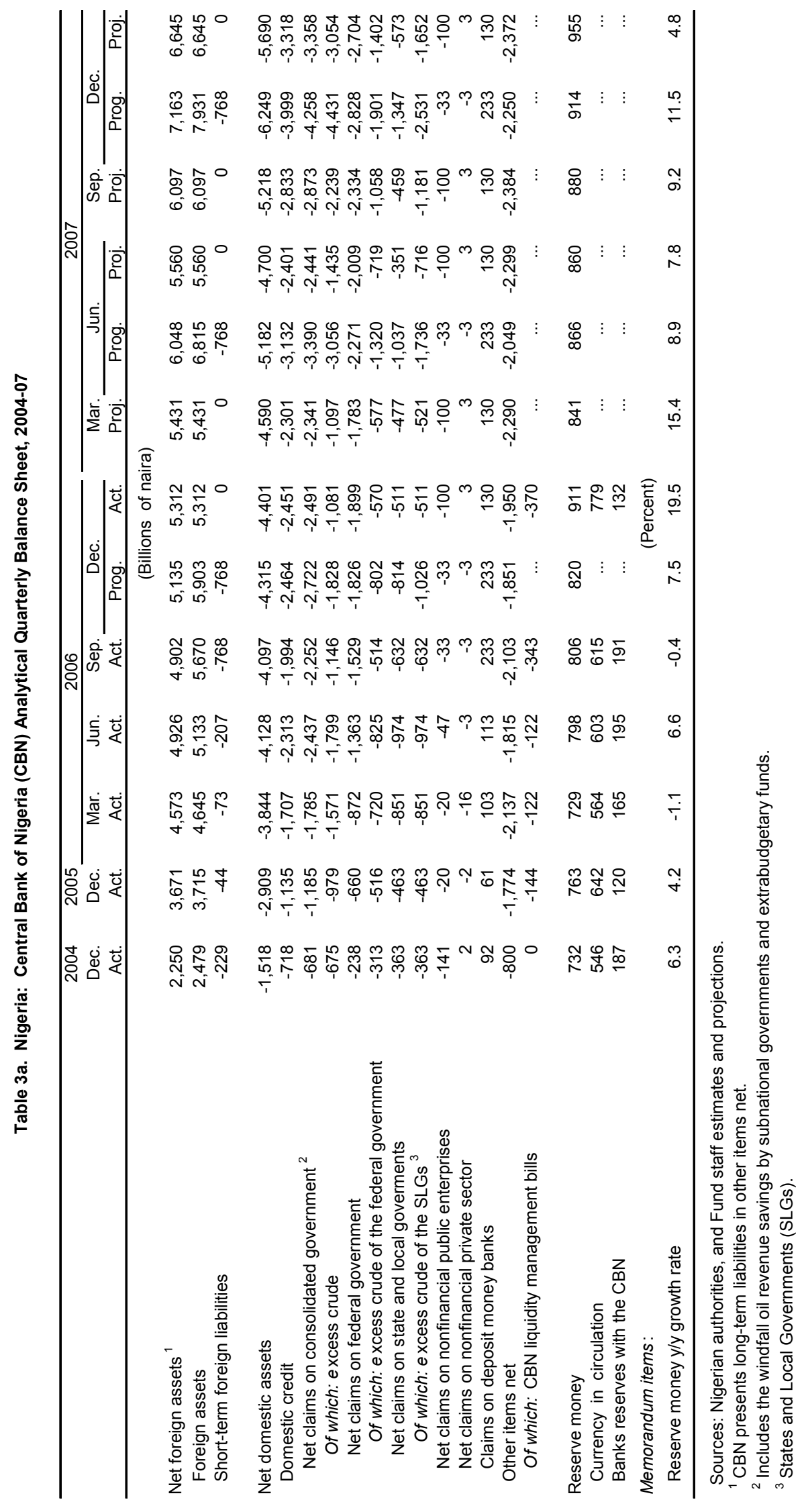




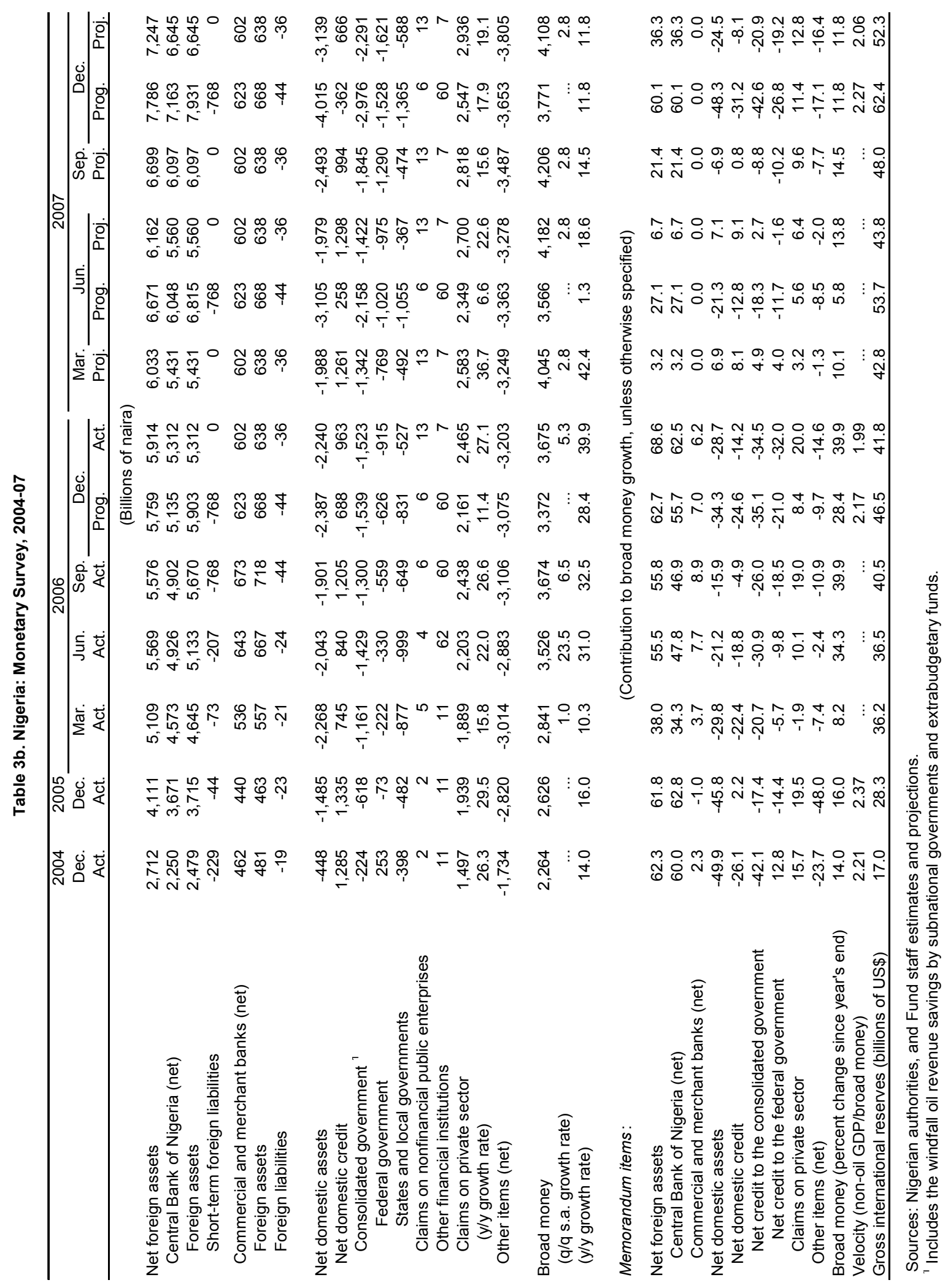


Table 4. Nigeria: Balance of Payments, 2004-10

(Billions of U.S. dollars, unless otherwise specified)

\begin{tabular}{|c|c|c|c|c|c|c|c|c|c|}
\hline & \multirow{2}{*}{$\begin{array}{r}2004 \\
\text { Act. }\end{array}$} & \multirow{2}{*}{$\begin{array}{r}2005 \\
\text { Act. }\end{array}$} & \multicolumn{2}{|c|}{2006} & \multicolumn{2}{|c|}{2007} & \multirow{2}{*}{$\begin{array}{r}2008 \\
\text { Proj. }\end{array}$} & \multirow{2}{*}{$\begin{array}{r}2009 \\
\text { Proj. }\end{array}$} & \multirow{2}{*}{$\begin{array}{r}2010 \\
\text { Proj. }\end{array}$} \\
\hline & & & Prog. & Est. & Prog. & Proj. & & & \\
\hline Current account balance & 3.8 & 9.1 & 14.4 & 13.6 & 13.6 & 8.9 & 10.8 & 9.7 & 14.1 \\
\hline Oil/gas (net) & 16.0 & 24.1 & 31.7 & 31.5 & 35.5 & 33.8 & 37.7 & 39.6 & 45.3 \\
\hline Other (net) & -12.1 & -15.0 & -17.3 & -17.9 & -21.9 & -24.9 & -26.9 & -29.8 & -31.2 \\
\hline Trade balance & 17.8 & 27.8 & 28.3 & 30.8 & 27.0 & 24.6 & 28.1 & 26.8 & 31.3 \\
\hline Exports & 37.3 & 53.1 & 58.7 & 61.6 & 63.7 & 63.6 & 70.2 & 72.0 & 78.5 \\
\hline Oil/gas & 36.4 & 52.4 & 57.9 & 60.8 & 62.8 & 62.7 & 69.3 & 71.1 & 77.4 \\
\hline Other & 0.9 & 0.7 & 0.8 & 0.8 & 0.8 & 0.9 & 0.9 & 1.0 & 1.0 \\
\hline Imports & -19.4 & -25.4 & -30.4 & -30.8 & -36.6 & -39.0 & -42.1 & -45.2 & -47.2 \\
\hline Oil/gas & -8.9 & -11.7 & -13.1 & -13.1 & -13.8 & -13.7 & -15.0 & -15.0 & -15.5 \\
\hline Other & -10.5 & -13.7 & -17.3 & -17.7 & -22.8 & -25.3 & -27.2 & -30.2 & -31.7 \\
\hline Services (net) & -5.9 & -6.7 & -5.9 & -5.9 & -6.4 & -6.5 & -8.2 & -8.4 & -8.8 \\
\hline Receipts & 1.7 & 2.0 & 3.4 & 3.4 & 3.5 & 3.5 & 2.5 & 2.7 & 2.9 \\
\hline Oil/gas & 0.3 & 0.5 & 1.7 & 1.8 & 1.8 & 1.8 & 0.6 & 0.6 & 0.7 \\
\hline Other & 1.3 & 1.5 & 1.6 & 1.6 & 1.7 & 1.8 & 1.9 & 2.0 & 2.2 \\
\hline Payments & -7.6 & -8.7 & -9.3 & -9.3 & -9.9 & -10.0 & -10.7 & -11.1 & -11.7 \\
\hline Oil/gas & -2.6 & -3.0 & -3.1 & -3.1 & -3.3 & -3.3 & -3.5 & -3.4 & -3.4 \\
\hline Other & -5.0 & -5.7 & -6.1 & -6.2 & -6.6 & -6.7 & -7.2 & -7.7 & -8.3 \\
\hline Income (net) & -10.9 & -15.3 & -11.4 & -14.6 & -10.4 & -12.6 & -12.6 & -12.1 & -11.9 \\
\hline Oil/gas & -9.3 & -14.1 & -11.7 & -14.8 & -11.9 & -13.7 & -13.8 & -13.7 & -13.9 \\
\hline Other & -1.6 & -1.3 & 0.3 & 0.2 & 1.5 & 1.1 & 1.2 & 1.7 & 2.1 \\
\hline Of which: Interest due on public debt & -1.5 & -1.6 & -0.3 & -0.2 & -0.1 & -0.2 & -0.2 & -0.2 & -0.2 \\
\hline Transfers (net) $^{1}$ & 2.8 & 3.4 & 3.4 & 3.4 & 3.4 & 3.4 & 3.4 & 3.4 & 3.4 \\
\hline Capital account balance & 2.7 & 5.3 & -4.7 & -2.7 & 2.3 & 2.1 & 8.5 & 8.5 & 8.6 \\
\hline Direct and portfolio investment (net) & 4.4 & 6.4 & 7.4 & 9.1 & 2.2 & 1.9 & 7.9 & 7.7 & 7.7 \\
\hline Oil/gas & 3.8 & 4.7 & 5.0 & 5.0 & 5.3 & 5.3 & 5.4 & 5.1 & 4.9 \\
\hline Other & 0.7 & 1.7 & 2.4 & 4.1 & -3.1 & -3.4 & 2.4 & 2.6 & 2.8 \\
\hline Official capital (net) & -1.3 & -1.2 & -4.6 & -4.5 & 0.2 & 0.2 & 0.7 & 0.8 & 0.9 \\
\hline Disbursements & 0.2 & 0.7 & 0.4 & 0.5 & 0.6 & 0.6 & 1.0 & 1.1 & 1.1 \\
\hline Amortization due & -1.5 & -1.9 & -5.0 & -5.0 & -0.5 & -0.4 & -0.4 & -0.3 & -0.3 \\
\hline Private borrowing (net) & 0.0 & 0.0 & 0.0 & 0.0 & 0.0 & 0.0 & 0.0 & 0.0 & 0.0 \\
\hline Other capital (net) ${ }^{2}$ & -0.4 & 0.1 & -7.5 & -7.3 & 0.0 & 0.0 & 0.0 & 0.0 & 0.0 \\
\hline Errors and omissions & 1.6 & 1.9 & -1.8 & 0.0 & 0.0 & 0.0 & 0.0 & 0.0 & 0.0 \\
\hline Overall balance & 8.1 & 16.3 & 8.0 & 10.9 & 16.0 & 11.0 & 19.3 & 18.3 & 22.6 \\
\hline Net international reserves (increase -) & -9.5 & -11.3 & -12.5 & -13.9 & -16.0 & -10.5 & -19.3 & -18.3 & -22.6 \\
\hline Exceptional financing & 1.4 & -4.9 & 4.5 & 3.0 & 0.0 & -0.5 & 0.0 & 0.0 & 0.0 \\
\hline Net accumulation of arrears (decrease -) & 1.3 & -5.4 & 4.5 & 3.0 & 0.0 & -0.5 & 0.0 & 0.0 & 0.0 \\
\hline Other $^{3}$ & 0.1 & 0.5 & 0.0 & 0.0 & 0.0 & 0.0 & 0.0 & 0.0 & 0.0 \\
\hline \multicolumn{10}{|l|}{ Memorandum items: } \\
\hline Gross official reserves, end-of-period & 17.0 & 28.3 & 46.5 & 41.8 & 62.4 & 52.3 & 71.6 & 89.9 & 112.5 \\
\hline In months of next year's GS imports & 6.0 & 8.5 & 12.0 & 10.2 & 14.2 & 11.9 & 15.3 & 18.3 & 21.6 \\
\hline Current account (percent of GDP) & 5.3 & 9.2 & 12.5 & 11.8 & 10.4 & 7.0 & 7.4 & 6.0 & 7.9 \\
\hline GS exports (percent of GDP) & 54.4 & 55.9 & 53.6 & 56.3 & 51.4 & 52.8 & 49.7 & 46.2 & 45.7 \\
\hline GS imports (percent of GDP) & 37.7 & 34.6 & 34.2 & 34.8 & 35.6 & 38.6 & 36.1 & 34.8 & 33.0 \\
\hline External debt ${ }^{4,5}$ & 35.9 & 20.5 & 3.5 & 3.5 & 3.7 & 3.3 & 3.9 & 4.7 & 5.6 \\
\hline External debt (percent of GDP) ${ }^{4,5}$ & 50.2 & 20.8 & 3.0 & 3.1 & 2.8 & 2.6 & 2.7 & 2.9 & 3.1 \\
\hline External debt (percent of GS exports) ${ }^{4,5}$ & 92.3 & 37.2 & 5.7 & 5.5 & 5.5 & 4.9 & 5.4 & 6.3 & 6.9 \\
\hline External debt ${ }^{4,5,6}$ & 116.9 & 47.8 & 6.7 & 7.0 & 6.1 & 5.9 & 6.3 & 7.1 & 7.8 \\
\hline External debt service due (percent of GS exports) & 7.8 & 6.4 & 8.5 & 8.0 & 0.9 & 0.8 & 0.7 & 0.6 & 0.5 \\
\hline GDP (at market prices) & 71.5 & 98.6 & 115.8 & 115.4 & 130.6 & 127.0 & 146.5 & 161.6 & 178.1 \\
\hline
\end{tabular}

Sources: Nigerian authorities, and Fund staff estimates and projections.

${ }^{1}$ Includes capital transfers.

${ }^{2}$ In 2006, the prepayment of post-cutoff Paris Club debt ( $\$ 0.3$ billion) and the cost of the buy-back of remaining Paris Club debt ( $\$ 4.3$ billion).

${ }^{3}$ In 2002 debt buy-back, in 2003-05 recovery of looted funds.

${ }^{4}$ Nominal public sector short- and long-term debt, end of period.

${ }^{5}$ In 2005 (2006) reflecting also a $\$ 7.1$ billion ( $\$ 7.2$ billion) write-off of Paris Club debt, and in 2006, reflecting the discount ( $\$ 2.7$ billion) on the $\$ 7.0$ billion buy-back of remaining Paris Club debt.

${ }^{6}$ Percent of general government fiscal revenues. 
Table 5. Nigeria: Non-oil Savings-Investment Balances, 2004-09 ${ }^{1}$

(Percent of non-oil GDP)

\begin{tabular}{|c|c|c|c|c|c|c|c|c|}
\hline & \multirow{2}{*}{$\begin{array}{r}2004 \\
\text { Act. }\end{array}$} & \multirow{2}{*}{$\begin{array}{c}2005 \\
\text { Act. }\end{array}$} & \multicolumn{2}{|c|}{2006} & \multicolumn{2}{|c|}{2007} & \multirow{2}{*}{$\begin{array}{c}2008 \\
\text { Proj. }\end{array}$} & \multirow{2}{*}{$\begin{array}{c}2009 \\
\text { Proj. }\end{array}$} \\
\hline & & & Prog. & Est. & Prog. & Proj. & & \\
\hline Income & 103.1 & 104.3 & 106.2 & 105.9 & 107.0 & 106.4 & 105.7 & 105.4 \\
\hline Public & 14.6 & 13.1 & 12.4 & 12.6 & 12.7 & 12.6 & 12.6 & 13.2 \\
\hline Private & 88.5 & 91.2 & 93.8 & 93.3 & 94.2 & 93.8 & 93.1 & 92.3 \\
\hline Consumption & 104.2 & 103.2 & 102.4 & 102.9 & 104.6 & 107.9 & 104.0 & 102.7 \\
\hline Public & 36.2 & 38.4 & 36.0 & 38.8 & 34.8 & 35.7 & 33.9 & 35.1 \\
\hline Private & 68.0 & 64.8 & 66.4 & 64.1 & 69.7 & 72.2 & 70.0 & 67.5 \\
\hline Savings & -1.1 & 1.1 & 3.8 & 3.1 & 2.4 & -1.5 & 1.7 & 2.8 \\
\hline Public & -21.6 & -25.3 & -23.6 & -26.2 & -22.1 & -23.1 & -21.3 & -22.0 \\
\hline Private & 20.4 & 26.4 & 27.4 & 29.2 & 24.5 & 21.7 & 23.0 & 24.7 \\
\hline Investment & 30.0 & 31.4 & 32.9 & 32.9 & 33.5 & 34.3 & 35.0 & 34.6 \\
\hline Public & 12.4 & 15.0 & 16.6 & 16.3 & 16.4 & 16.8 & 17.5 & 16.8 \\
\hline Private & 17.6 & 16.4 & 16.3 & 16.6 & 17.1 & 17.5 & 17.5 & 17.8 \\
\hline Current account & -31.1 & -30.3 & -29.0 & -29.8 & -31.1 & -35.8 & -33.3 & -31.8 \\
\hline Public & -33.9 & -40.3 & -40.2 & -42.5 & -38.5 & -39.9 & -38.8 & -38.8 \\
\hline Private & 2.8 & 10.0 & 11.1 & 12.6 & 7.4 & 4.2 & 5.5 & 7.0 \\
\hline \multicolumn{9}{|c|}{ Memorandum items: (percentage change) } \\
\hline Real private demand & 4.1 & 3.0 & 10.0 & 8.2 & 12.0 & 19.4 & 4.0 & 3.8 \\
\hline Real private consumption & 2.2 & 3.5 & 11.1 & 7.7 & 11.9 & 21.0 & 3.4 & 2.7 \\
\hline Real private investment & 12.3 & 1.1 & 5.8 & 10.2 & 12.2 & 13.4 & 6.5 & 8.3 \\
\hline
\end{tabular}

Sources: Nigerian authorities, and Fund staff estimates and projections.

' This table uses fiscal data on a commitment basis. 


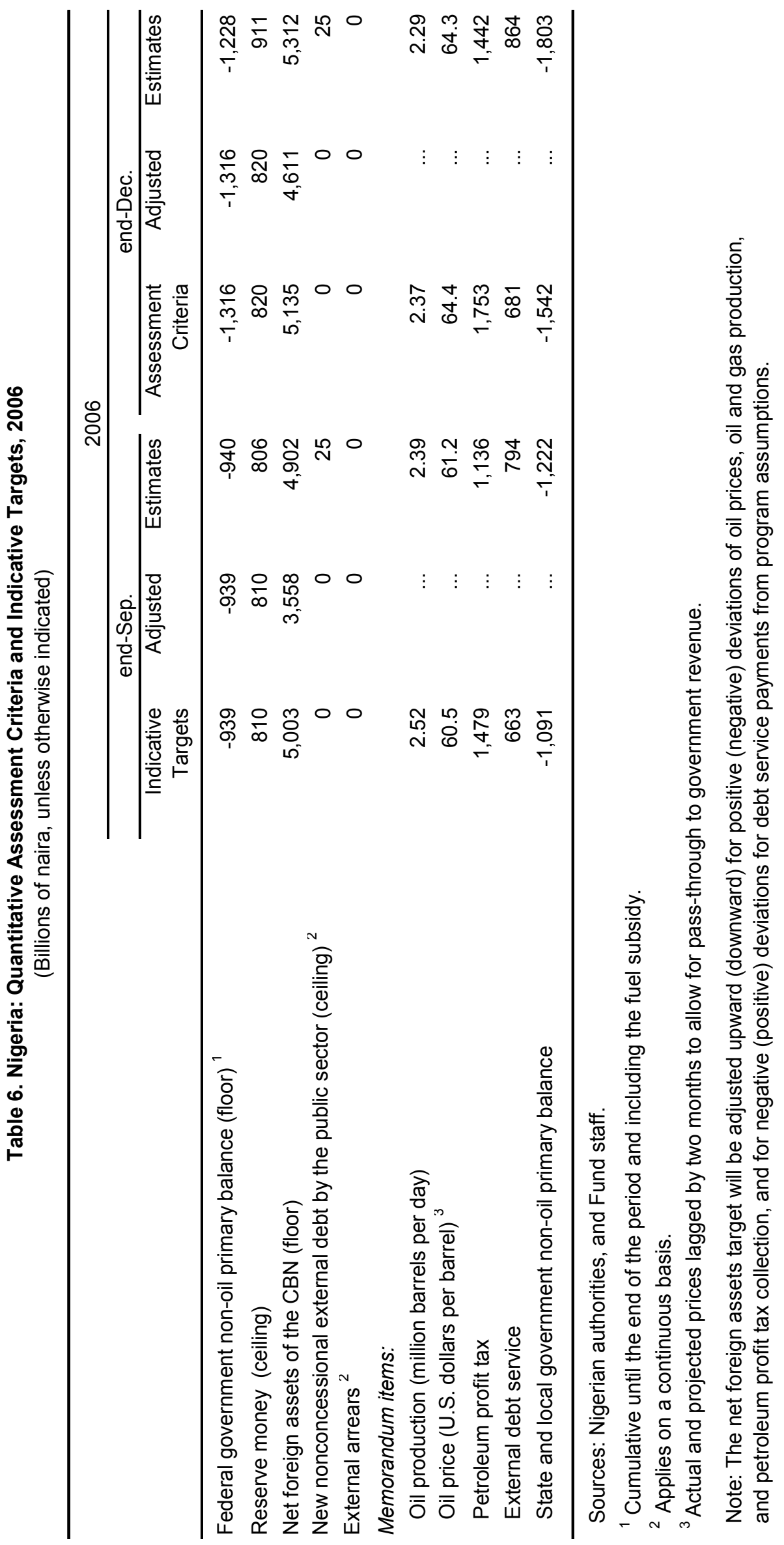




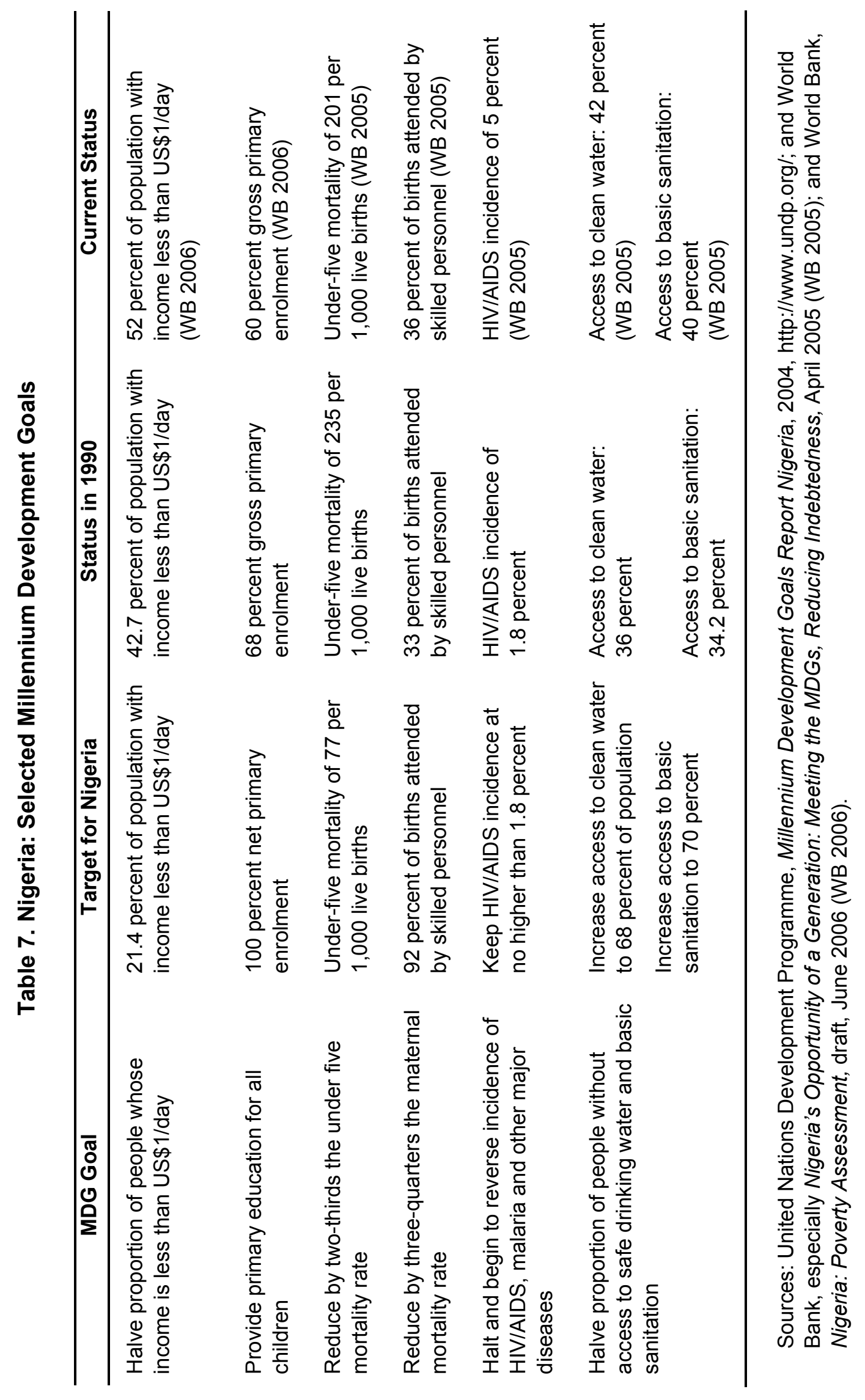


Table 8a. Nigeria: Structural Assessment Criteria for the 3rd Review, and Structural Benchmarks until April 2007

\begin{tabular}{lccc}
\hline $\begin{array}{l}\text { Structural Assessment Criteria and } \\
\text { Benchmarks }\end{array}$ & $\begin{array}{c}\text { Policy } \\
\text { Statement }\end{array}$ & $\begin{array}{c}\text { Expected } \\
\text { Date of } \\
\text { Achievement }\end{array}$ & Status \\
& & Description \\
\hline
\end{tabular}

Assessment Criteria

Introduce Integrated Personnel and Mar 2006 Sep 2006

Payroll Information System (IPPIS).

Establish database of pensioners and estimate size of pension arrears .

FIRS to conduct nationwide taxpayer enumeration in preparation for introducing automated tax administration system, including TIN as set out in paragraph 5 of the statement.

The Central Bank of Nigeria to establish prudential standards for consolidated supervision and begin to supervise the banking groups on a consolidated basis.

Management contract for the Transmission company of Nigeria will be awarded as set out in paragraph 9 of the statement

Appoint auditors to conduct the audit of the oil and gas sector for 2005 and 2006 as set out in paragraph 9 of the statement.

Structural Benchmarks Continue publication of revenue allocation to the three tiers of Government.

Financial bid opening of Port Harcourt and Kaduna refineries.

Produce quarterly report of spending in MDG-related sectors (Health, Education, Power, Water, Roads, and Agriculture) to cover Q1 and Q2 in the first instance.

Finalize and issue procurement manual.

Mar 2006

Sep 2006

Mar 2006 Dec 2006

Mar 2006 Dec 2006

Dec 2006 Mar 2007

Dec 2006 Mar 2007

Mar 2006 Jun 2006

Mar 2006 Sep 2006
Not met. Software installation in the 6 ministries and 6 central agencies of the $1 \mathrm{st}$ phase completed in March. Test payroll processing with the new system commenced in April, and deployment expected soon.

Not met.

Scale of necessary work was expanded and expected observed by July 2007. Delay due to an unanticipated second exercise to verify basic taxpayer information.

Not met. Remedial measures include a time-bound action plan by the time of the fourth review, and plans for a longterm technical assistance expert from mid-2007. Unanticipated problems related to post-consolidation of banks emerged and precluded the immediate implementation of consolidated supervision.

Not met. Expected observed by end-May. Delays were caused by procedure requirements in this World Bank sponsored project. Expressions of interest were evaluated, and bid documents issued to four prequalified companies.

Not met. Observed for 2005 audit. Delay with contract unlikely to hold up the 2006 audit. For the 2006 audit, open procurement has been launched.

Oct 2005 Continuous Observed. Data are being published regularly on the MoF website at http://www.fmf.gov.ng/detail.php?link=faac.

Not met. Negotiations with interested bidders resumed now that draft sector legislation prepared. Kaduna Refinery offered as an integrated package to CNPC of China. For Port Harcourt, eight expressions of interest received.

Mar 2006 Sep $2006 \quad$ Not met. Preparation of quarterly reports on debt relief expenditures commenced, but the reports are yet to be automated for systematic data generation, because of delays in full implentation of the computerized accounting system ATRRS in the OAGF.

Not met. Draft manual prepared that can be finalized and issued once the Procurement Bill is passed. 
Table 8a. Nigeria: Structural Assessment Criteria for the 3rd Review, and Structural Benchmarks until April 2007, (concluded)

\begin{tabular}{|c|c|c|c|c|}
\hline $\begin{array}{l}\text { Structural Assessment Criteria and } \\
\text { Benchmarks }\end{array}$ & $\begin{array}{c}\text { Policy } \\
\text { Statement }\end{array}$ & $\begin{array}{c}\text { Expected } \\
\text { Date of } \\
\text { Achievement }\end{array}$ & Status & Description \\
\hline
\end{tabular}

Structural Benchmarks (continued)

Conduct transparency and anticorruption survey in at least six pilot states.

Set up a tax policy unit in the Ministry of Finance.

Settle contractor arrears in cash for Mar 2006 creditors with claim of up to N100 million and issue bonds to cover 50 percent of debts owed to larger creditors.

Continue reform of Nigeria Mar 2006 Dec 2006 Customs Service by expanding the operations of the Large Importers/Exporters Unit to handle at least 50 percent of the trade.

Complete payment of severance benefits and training programs for retirees resulting from the Civil Service reform program in MDAs.

Mar 2006 Dec 2006

Dec 2006
Mar 2006 Dec 2006

\section{with delay.}

All verified pre-2005 contractors' arrears have been paid. Regarding arrears of less than N100 million, the first wave of claims was settled by end-December. However, some previously denied claims had to be settled in a second wave that was only concluded in March 2007. While it may be necessary to assess a third wave of claims, it is expected that all legitimate claims have already been paid. Regarding large contractors' claims, bonds were issued as the legitimacy of claims was being established; all large contractor arrears have now been settled.

Not met. Classification of traders into fast-track channels - the blue channel for large importers/ exporters, and the green channel for pre-qualified traders - has been expanded, but so far only about 30 - 40 percent of trade is covered. Delays are caused by maintaining appropriate safeguards.

Dec 2006 Mar 2007

Not met. More than 30,000 officers have completed retraining but as of mid-April payment of severance benefits for about 3,000 officers was pending, because of required verification by the Auditor General that the disengaged civil servant does not have outstanding obligations to the government. This structural benchmark only refers to the first phase of disengagements, that identified appoximately 30,000 officers without the relevant skills for employment in the public service. Additional disengagements (so far 10,000 from the core civil service, and 11,000 from parastatals) are taking place as agencies are being restructured, and of these 10,000 officers have already received their retraining.

Complete restructuring of 11 core

Dec 2006 Mar 2007 Ministries as set out in paragraph 9 of the statement.
Observed. 12 ministries in the delineation in effect until January 2007 were reorganized by end-March. For five of these ministries some additional restructuring will be necessary to complete the merger with other ministries that was initiated in January 2007. 
Table 8b. Nigeria: Structural Assessment Criteria for the 4th Review, and Structural Benchmarks for May - June, 2007

\begin{tabular}{|c|c|c|c|c|}
\hline $\begin{array}{l}\text { Structural Assessment Criteria and } \\
\text { Benchmarks }\end{array}$ & $\begin{array}{c}\text { Policy } \\
\text { Statement }\end{array}$ & $\begin{array}{c}\text { Expected } \\
\text { Date of } \\
\text { Achievement } \\
\end{array}$ & Status & Description \\
\hline \multicolumn{5}{|l|}{ Structural Assessment Criteria } \\
\hline $\begin{array}{l}\text { Complete restructuring of MDAs as } \\
\text { set out in paragraph } 9 \text { of the } \\
\text { statement. }\end{array}$ & Dec 2006 & May 2007 & Pending. & $\begin{array}{l}12 \text { ministries, departments and agencies (MDAs) } \\
\text { restructured by end-March. Significant preparatory work } \\
\text { for the restructuring of all MDAs expected completed by } \\
\text { end-May, but process now expected to be finalized by } \\
\text { the new administration. }\end{array}$ \\
\hline $\begin{array}{l}\text { Bid opening for sale of the Abuja } \\
\text { Electricity Distribution Plc as set } \\
\text { out in paragraph } 9 \text { of the } \\
\text { statement. }\end{array}$ & Dec 2006 & Jun 2007 & On track. & $\begin{array}{l}\text { Expressions of interest for the Abuja Electricity } \\
\text { Distribution Plc have closed. }\end{array}$ \\
\hline $\begin{array}{l}\text { Issue report on SEEDS } \\
\text { Benchmarking for } 36 \text { States to be } \\
\text { published as set out in paragraph } 9 \\
\text { of the statement. }\end{array}$ & Dec 2006 & Jun 2007 & On track. & $\begin{array}{l}\text { Draft report on the results of the } 2006 \text { SEEDS } \\
\text { benchmarking under review and expected to be } \\
\text { published in Q2 } 2007 \text {. }\end{array}$ \\
\hline $\begin{array}{l}\text { FIRS to complete implementation } \\
\text { of human resource management } \\
\text { system, including installation of } \\
\text { HRM hardware and software for } \\
\text { nationwide access, and conduct a } \\
\text { competence assessment exercise } \\
\text { to determine training needs. }\end{array}$ & Dec 2006 & Jun 2007 & Pending. & $\begin{array}{l}\text { Preparations for the competency assessement and } \\
\text { staff training underway. Regarding the introduction of } \\
\text { the human resource management system, the } \\
\text { consultant had to be replaced, likely delaying the } \\
\text { project. }\end{array}$ \\
\hline $\begin{array}{l}\text { The Central Bank to introduce a } \\
\text { new Standing Facility to help } \\
\text { reduce interest rate volatility as } \\
\text { stated in paragraph } 7 \text { of the } \\
\text { statement. }\end{array}$ & Dec 2006 & Jun 2007 & Observed. & The facility was introduced in mid-Dec 2006. \\
\hline \multicolumn{5}{|l|}{$\underline{\text { Structural Benchmarks }}$} \\
\hline $\begin{array}{l}\text { Complete restructuring of five } \\
\text { parastatals in terms of right-sizing } \\
\text { and right-staffing. }\end{array}$ & Dec 2006 & May 2007 & On track. & $\begin{array}{l}\text { The reorganization of the CBN, NBS, and Center for } \\
\text { Management Development has been completed, and } \\
\text { the reorganizations of NNPC and FIRS are at an } \\
\text { advanced stage. The reorganization of additional } \\
\text { parastatals is also underway. }\end{array}$ \\
\hline $\begin{array}{l}\text { Bid opening for sale of } 8 \text { Oil } \\
\text { Service Companies. }\end{array}$ & Dec 2006 & Jun 2007 & Observed. & $\begin{array}{l}\text { The } 8 \text { companies were sold in January as the } \\
\text { negotiations proceeded more smoothly than expected. }\end{array}$ \\
\hline $\begin{array}{l}\text { Nigerian Customs Service to } \\
\text { conduct staff survey to determine } \\
\text { suitability of personnel for minimum } \\
\text { requirements of service in } \\
\text { preparation for rationalization, } \\
\text { realignment and right-sizing its } \\
\text { human resource system. }\end{array}$ & Dec 2006 & Jun 2007 & Observed. & $\begin{array}{l}\text { The staff survey has been conducted, as well as a } \\
\text { competency assessment; the draft report is with } \\
\text { management. }\end{array}$ \\
\hline
\end{tabular}




\section{Appendix I. The Authorities' Letter}

Mr. Rodrigo de Rato

Managing Director

International Monetary Fund

$70019^{\text {th }}$ Street N.W.

Washington, DC 20431

Dear Mr. de Rato:

The attached Policy Statement describes economic and financial policies that the government of Nigeria has been pursuing and wants to continue to implement. The Policy Statement outlines our broad macroeconomic objectives and policies for the remainder of 2007 and for the medium term. These policies are based on our National Economic Empowerment and Development Strategy (NEEDS).

2. The Government of Nigeria believes that the policies set forth in the attached statement are adequate to achieve the objectives of our PSI program. Given our interests in macroeconomic stability, we stand ready to take additional measures as may be necessary to achieve needed objectives. Our Policy Statement requests modification of assessment criteria for end-June 2007 for the fourth review expected to be considered by the Executive Board of the IMF by October 16, 2007 to reflect recent macroeconomic performance. We stand ready to work with the Fund and the Bank in partnership in the implementation of our home grown program and will naturally consult in advance should revisions be contemplated to the policies contained in the PSI.

/sgd/

Mrs. Nenadi E. Usman

Minister of Finance
$/ \operatorname{sgd} /$

Professor Chukwuma C. Soludo

Governor, Central Bank of Nigeria 
Attachment I. Policy Statement by the Government of Nigeria on the Fourth Phase of the Nigerian Economic Reform Program Under the Policy Support Instrument

May 2007

This policy statement by the Government of Nigeria is on the fourth and final phase of Nigeria's Policy Support Instrument (PSI) following the conclusion of the first three Reviews of the PSI by the IMF. It summarizes recent developments on the reforms, including progress with the existing structural assessment criteria and benchmarks, and the short-and medium-term objectives going forward. Some of the quantitative and structural targets were missed but the overall strength and direction of the program remain very positive.

1. This Policy Statement by the Government is on the fourth and final phase of the Policy Support Instrument (PSI), following earlier Statements dated October 6, 2005, March 2006, and December 2006. The Statement spells out the Government's economic reform program covering macroeconomic, structural and anti-corruption issues. The third review mission took place during February 14 to 27, 2007. Nigeria's reform program, which was instituted by the Government of President Obasanjo in 2003, and based on the country's home-grown poverty reduction program, the National Economic Empowerment and Development Strategy (NEEDS), focuses on addressing the structural and institutional weaknesses of the economy, tackling corruption and overhauling the public expenditure management. To ensure an objective assessment of the implementation of the program, the IMF has been monitoring on a quarterly basis since 2004 the implementation of the program at the invitation of the Government within the context of intensified surveillance.

2. The broad goals of NEEDS are poverty reduction, wealth creation and employment generation through the development of an enabling environment in the form of fiscal discipline through an oil price-based fiscal rule, improved public expenditure management, better public procurement systems and better service delivery combined with more efficient revenue collection, tax reforms and customs reforms. Progress in these areas has provided a stable macroeconomic environment, which should enable the private sector to play its role as the engine of economic growth. Similarly, the anti-corruption drive of the Economic and Financial Crimes Commission (EFCC), and the work of the Nigerian Extractive Industries Transparency Initiative (N-EITI) in auditing the oil and gas industry are addressing lapses in governance and transparency within that sector. Furthermore, ongoing reforms in the public service would bring about efficiency, just as market liberalization and privatization of key public enterprises are helping to improve the public finances and quality of service. Finally, following the adoption of a Wholesale Dutch Auction System after the banking consolidation, the exchange rates on foreign exchange markets have converged since mid-2006.

3. The reforms have continued to deliver significant results. Macroeconomic stability has resulted in growth, while other key indicators have also been positive. In 2006, real GDP grew by a robust 5.6 percent in spite of a 4.7 percent decline in oil GDP due to disruptions to production in the Niger Delta. Non-oil GDP grew by 8.9 percent. This follows the growth rates 
of 6.5 percent and 6.6 percent recorded in 2005 and 2004 respectively. In all three years, the performance of the non-oil sector dominated real GDP growth; in 2004 and 2005, for example, non-oil GDP grew by 7.8 percent and 8.6 , respectively.

4. The performance of the fiscal sector has also been impressive. The consolidated fiscal surplus for 2006 was about 9 percent of GDP, following the surpluses of 11 percent and 10 percent of GDP recorded in 2005 and 2004 respectively. The balance on the excess crude oil account as of end December 2006 stood at $\$ 8.3$ billion and N627.5 billion (the latter reflecting the balance for the domestic crude oil component). Foreign reserves as of end 2006 rose to $\$ 42.3$ billion (from $\$ 28.3$ billion a year earlier) as a result of continued prudent fiscal policy with support from monetary policy. Inflation has continued on a declining path; at end December, the rate was 8.5 percent (year on year) compared to 11.6 percent at end 2005, reflecting mainly the effect of good harvest, as foodstuff constitutes about $64 \%$ percent of the CPI basket. The 12-month average inflation rate was 8.2 percent. The reserve money target was exceeded by about 11 percent, largely reflecting the effect of the unexpected growth in currency in circulation in the fourth quarter, influenced by seasonal factors as well as pre-election activities by the various political parties during the period. The CBN successfully introduced a new Monetary Policy Rate (MPR) in December 2006 designed to address the problem of interest rate volatility; preliminary data indicate that this has started to have a calming effect on the rates.

5. Following the exit from the Paris Club, Nigeria is now working to clear its London Club debt; it has redeemed the Par Bonds ( $\$ 1.486$ billion) and repurchased its promissory Notes; it has already successfully repurchased about $1 / 3$ of its Oil Warrants. As of end-2006, Nigeria's external debt stood at about $3 \%$ of GDP, most of this being multilateral debt. Two international rating agencies (Fitch and Standard \& Poor's) undertook the first ever sovereign ratings for the country in 2005 (both with a rating of BB- with stable outlook). The 2006 rating exercise by both confirmed the 2005 rating. Building on the two successful PSI reviews of end-December 2005 and end-June 2006 targets, Nigeria has met a significant number of the targets agreed for the period up to end-March 2007 and is on course to meet most of those set for May/June 2007. Indeed, some of the assessment criteria and structural benchmarks have already been met (see Table 2). The targets for the $4^{\text {th }}$ phase of the PSI program are as shown in tables 1 and 2.

\section{The 2007 budget was passed by the National Assembly and signed into law in} December 2006, the first time this has happened in advance of the commencement of the new fiscal year. The budget was based on an average price of $\$ 40$ per barrel for Nigerian crude oil, following the price-based fiscal rule that has been in operation since 2004 . The strategy entails building on the gains of the last three years, continued adherence to the medium term expenditure framework (MTEF), prudence in public expenditure management and observance of due process in public procurement. Other aspects include the intensification of the fight against corruption, and a continuation of the privatization exercise, including deregulation of key sectors, extending the reforms to the rest of the public sector and strengthening of trade facilitation. With the attainment of macroeconomic stability, the Government is now focusing on employment generation through the removal of bottlenecks to private business, promoting real sector activities 
(including through a more vigorous implementation of the local content policy), human capital development, and improved service delivery. In a related context, the Government is laying increasing emphasis on the development of the nation's infrastructure in part, by drawing on the accumulated oil savings to fund a number of important projects. In implementing these projects, the Government aims to safeguard macroeconomic stability, including selecting projects with high import content, and to ensure a high quality of spending. ${ }^{1}$ The short- and medium-term macroeconomic framework and financial policies remain broadly as in the original PSI statement, as summarized below:

\section{Short-Term Macroeconomic Policies}

\section{Short-term macroeconomic policy will revolve around the implementation of the} 2007 budget, including the extra expenditure on infrastructure. It is recognized that in order to enable the private sector to successfully play its role as the main driver of economic growth, infrastructure needs to be strengthened. ${ }^{2}$ In this regard, the Government is increasing investment in roads, railways, electricity, and in the dredging of the River Niger to facilitate transportation through the inland waterways. An estimated 75-80 percent of the expenditure will be in the form of imports and part of it will be funded from the excess crude oil account. Towards ensuring good quality capital spending and effective use of excess crude oil account, Government organized a workshop for key stakeholders in February in collaboration with the IMF, World Bank, Government of Norway and other development partners on the theme, "Infrastructure Gap and Management of Oil Savings in Nigeria: Issues and Challenges". The outcome document from the workshop provides a roadmap that will partly guide Government in these areas. An action plan for the implementation of the recommendations will be developed by the Government. The oversight role of the Due Process Office will continue to underpin the effort of Government to ensure that the quality of spending is not compromised. In order to ensure that the liquidity impact of the extra spending is managed properly, interaction between the fiscal and monetary authorities are being intensified to enable the Central Bank have up-to-date information on the domestic component of this spending so it can take timely action in the management of the liquidity impact of the extra spending. A Fiscal Liquidity Assessment Committee (FLAC) comprising of representatives of the Ministry of Finance, Budget Office, CBN, OAGF, NNPC, and FIRS has been set up to monitor expenditure developments and their liquidity implications. The FLAC now hold weekly meetings to review developments and to provide advice. Similarly, provision has been made in the budget to fund the cost of liquidity mop-up. Going forward, specific elements of the macroeconomic framework include the following:

\footnotetext{
${ }^{1}$ In a related move towards ensuring high quality spending, the Government in collaboration with the IMF and World Bank, organized a workshop on "The Infrastructure Gap and Oil Savings: Issues and Challenges" during the $3^{\text {rd }}$ Review mission.

${ }^{2}$ The private sector in Nigeria has consistently flagged poor infrastructure as a drawback to business activity, confirming the findings of several studies in this regard.
} 
- Crude oil production is projected to average $2.50 \mathrm{Mb} / \mathrm{d}$ in 2007 , taking into account the gradual recovery from the disruption to production in the Niger Delta.

- An oil price-based fiscal rule will continue to underpin the budgeting process; for the 2007 budget, the exact benchmark price was $\$ 40$ per barrel.

- The targeted primary expenditure of the Federal Government in 2007 is estimated at about 10.9 percent of GDP. In the light of the planned higher capital spending, and the fact that a new Government will be taking office from May, Nigeria will still strive to attain the programmed consolidated non-oil primary deficit of 42 percent of non-oil GDP in 2007 (also taking account of the fuel subsidy), including by careful management of spending of the overflow of the 2006 capital budget, and by prudent implementation of the 2007 budget. However, it should be pointed out that the 2007 budget does not take account of any changes that might occur as a result of the transition to a new Government. Similarly, the Government expects to continue to implement the structural targets set in the 2 nd review (see Table 2).

- $\quad$ Excess revenue on the crude oil account will continue to be saved. It is estimated that the balance on the excess crude oil account would rise to the equivalent of 15.6 percent of GDP at end 2007 on the basis of current WEO oil price projections (of \$60.8 per barrel average for Nigerian oil in 2007) and a $\$ 40$ per barrel oil benchmark price.

- $\quad$ Based on the above, the stock of international reserves is expected to rise to $\$ 52.3$ billion at end $2007^{3}$.

- With continued prudent fiscal policy and a proactive monetary policy, inflation is estimated to remain in single digits, following the 8.2 percent level achieved at end 2006. Related to this, broad money growth rate of about 28 percent targeted for the year by the $\mathrm{CBN}$ was exceeded by about 12 percentage points. The $\mathrm{CBN}$ is committed to an exchange rate policy in line with the monetary policy objective of single-digit inflation.

- A real non-oil GDP growth rate in the range of 7-10 percent is being targeted by the Government for 2007, with the non-oil sector expected to continue to be the main driver.

- With all 36 States having developed their counterpart to the NEEDS program, the State Economic Empowerment and Development Strategy (SEEDS), Government is continuing to engage States with a view to deepening the reforms to the level of subnational Governments, particularly in the areas of public expenditure management, debt management, public procurement, tax reforms, and privatization. The passage of key

\footnotetext{
${ }^{3}$ It must be emphasized that the projections for the excess crude oil account and the external reserves are susceptible to the developments in the international oil market. Similarly, the same risk applies if actual oil production turns out to be below the projected level.
} 
legislation, such as the Fiscal Responsibility Bill and Public Procurement Bill will help to underpin the reforms generally, but the former will especially push the public expenditure reforms to the State level. Furthermore, emphasis is being given in the SEEDS implementation framework to the attainment of the MDGs. An assessment of the implementation of the NEEDS has been undertaken by the National Planning Commission, while an early draft of NEEDS-II has been completed and undergoing review. In this respect, some stakeholder retreats have been held to seek inputs from various groups.

- The monetary program continues to be based on zero net lending to the Government by the CBN although in exceptional circumstances, it would lend to the Government but keep such lending at no more than 5.0 percent of the previous year's retained revenue, and the loan must be cleared to zero by the end of the quarter.

- $\quad$ The $\mathrm{CBN}$ has introduced a new policy instrument, the monetary policy rate (MPR) to help reduce interest rate volatility. Combined with the role of the FLAC, the new framework will help to bring about a more effective control of the liquidity situation. Initial evidence shows that volatility has, indeed, started to decline.

- $\quad$ The CBN has made progress in its effort to introduce a system of consolidated supervision of banks. In this context, it has prepared a draft framework, which it is discussing with key stakeholders, including sister regulatory agencies (SEC, NAICOM and NDIC). Further, it hosted a workshop on consolidated supervision conducted by the Office of the Superintendent of Financial Institutions (OSFI), Canada under a technical assistance program during April 16-19, 2007. By the time of the fourth review of the PSI, there will be a time-bound action plan for the introduction of consolidated banking supervision.

- The CBN continues to strengthen its effectiveness through an ongoing internal restructuring program to refocus its work towards a more effective conduct of monetary policy.

- The CBN is on course in its restructuring of the Nigerian Security Printing and Minting (NSPM, Plc) and the four lower currency denominations (5, 10, 20, and 50 Naira) and the issuing of new coins ( 50 kobo, N1, and N2). These measures are expected to lead to a reduction in the cost of currency issuance.

\section{Medium-Term Macroeconomic Policy}

8. The implementation of the reform program is expected to continue in the medium term under the incoming Administration. The medium-term macroeconomic policy will continue to target macroeconomic stability and structural reforms. The framework will be based on the following assumptions: 
- Crude oil production is expected to continue to increase in the medium term, possibly reaching about $2.70 \mathrm{Mb} / \mathrm{d}$ in 2008 , and thereafter rise further in line with current Government plans to increase the production capacity. The oil price based fiscal rule will continue to underpin the budgeting process, particularly with the recent passage of the Fiscal Responsibility Bill by the National Assembly.

- The targeted primary expenditure of the Federal Government in 2008 is projected at about 10.9 percent of GDP.

- The monetary program in the medium term will continue as envisaged under the short term program with regard to broad monetary targets, and the new monetary policy framework, with priority continuing to be given to price stability.

9. Structural reforms are being deepened. Several public enterprises listed for privatization in 2006 and 2007 were successfully sold, including eight oil service companies, while others are on target for sale. The following enterprises are targeted for privatization in 2007:

- With the unbundling of the Power Holding Company of Nigeria (PHCN), Government is now working to privatize all eleven of the distribution companies. Work on the contract for the management of the Transmission Company of Nigeria is ongoing, but there are indications that there might be some delay due to slow procedures in the procurement process for World Bank sponsored projects. Expressions of interest have now been evaluated, and bid documents issued to the four pre-qualified investors. This is now expected to be completed by end-May 2007. Similarly, the bid opening for the Abuja Electrical Distribution Plc will be done by end-June 2007.

- $\quad$ Following initial delays, negotiations with interested bidders in the Kaduna and Port Harcourt refineries have resumed, following the preparation of the draft sector legislation. Kaduna Refinery has been offered as an integrated package to CNPC of China. In the case of Port Harcourt, eight expressions of interest have been received. It is expected they will be offered for sale in 2Q 2007.

- 8 oil service companies slated to be offered for sale in 2Q 2007 were successfully sold ahead of schedule (in January).

- 9 LPG plants will be offered for sale in 3Q 2007.

\section{Other structural reforms}

- Following the adoption of the five-band customs tariff in October 2005 under the Common External Tariff (CET) of the West African regional economic bloc, ECOWAS, the Government will now work towards reducing or eliminating the prohibition list (banned items) by a date that is consistent with the date for ECOWAS convergence 
criteria. The 50 percent tariff rate will be reviewed by end-2007, following a study on the impact of the tariff reform on the economy.

- The reform of the Nigeria Customs Service (NCS) continues. The staff survey has been conducted and a competency assessment has been done; the draft report is now being considered by management. Similarly, the drive to have more goods cleared through the fast track channel has continued to progress, with an estimated 30-40 percent of goods now passing through this channel. This is somewhat less than had been targeted because of a discovery that some of the beneficiaries might be taking undue advantage of the system.

- The National Planning Commission commenced the 2nd SEEDS benchmarking in November 2006, using independent assessors ${ }^{4}$ Civil Society organizations and other stakeholders were also brought into the process, including development partners. It is expected that the work will be completed soon with a view to publishing the outcome by the end of the second quarter of 2007.

- In order to strengthen the management of public accounts, the Government is currently computerizing the Budget Office and Office of the Accountant General of the Federation (OAGF), with a view to interconnecting their operations with those of the Budget Monitoring and Price Intelligence Unit (BMPIU) and CBN in order to reinforce the Accounting Transaction Recording and Reporting System (ATRRS). The ATRRS will be made consistent with the Integrated Financial Management System (IFMS) being developed with World Bank support.

- A Chart of Accounts has been developed by the Office of the Accountant General, and is now in full operation. The ATRRS is now being extended to Nigerian Missions/Embassies abroad for better financial control in this sector.

- Following the implementation of a tagging and tracking system for monitoring and evaluating spending of debt relief savings in MDG-related sectors, Government has now commenced the compilation of quarterly reports on the debt relief expenditure. However, it is yet to be automated for systematic data generation, because of delays in full implementation of the computerized accounting system ATRRS in the OAGF.

- $\quad$ Significant progress has been made in the payment of contractor arrears. The program commenced in July 2006; contractors that are owed up to N100 million have been fully paid off, while bonds have been issued to cover the debt to contractors owed over N100 million.

\footnotetext{
${ }^{4}$ The 2006 exercise involved 3.3 States compared to 35 in the maiden edition of the benchmarking. The other three States could not participate because of logistical difficulties.
} 
- Similarly, a database of pensioners has been established and the clearing of arrears is ongoing, with cash payment for arrears for 2005 and bonds for the pre-2005 arrears.

- $\quad$ The Debt Management Office (DMO) has made significant progress in its work towards clearing Nigeria's London debt. It successfully concluded the retirement of the par bonds by paying $\$ 1.486$ billion, and also the promissory notes and oil warrants. It has developed a debt management framework, which will guide future operations in the areas of borrowing, terms and conditions, repayment, etc. The framework is now being discussed with stakeholders so as to get their buy-in. The Fund has also reviewed the draft, and the Government will be requesting Fund technical assistance for further refinement of the document, after which it would be finalized.

- $\quad$ The work of the Due Process Office in ensuring transparency and best practice in public procurement is continuing; BMPIU has completed a draft of the procurement manual, which will be finalized and published once the Public Procurement Bill is passed into law.

- More progress has been made in respect of payments to depositors whose funds were trapped in the 14 banks that failed to meet the new capitalization threshold; final court orders have been obtained in respect of 10 of the banks; and a provision court order in respect of another, with three others still pending. The healthy banks have been invited to assume the private sector deposits of banks for which court orders have been granted and to cherry-pick the assets of these banks under the Purchase and Assumption resolution method.

- With the international price of oil in 2007 so far lower than in 2006, the amount of oil subsidy has declined, particularly after the upward adjustment of the ex-depot price of diesel in July, 2006. However, the Federal Government had to bear most of the subsidy as lower-tier Governments were unable to contribute their share of the agreed price support. At present international oil prices, fuel subsidy is minimal, limited mostly to kerosene (which is used mostly by the poor). Going forward, Government shall continue to work towards the liberalization of the sector.

- $\quad$ Progress continues to be made in respect of the recapitalization of Community banks (from N5 million to N20 million within a time frame of 18 months).

- $\quad$ The Civil Service reforms are continuing, with full disengagement of 30,000 staff who were not supposed to be in the service in the first place; payment of severance benefits and training for smooth transition to post-retirement life is nearly completed, with full training already provided by end March. Severance payments are progressing, but the process is somewhat slower because of a need to check that the departing staff do not have any outstanding debt to the Government.

- A new salary structure has been introduced designed to motivate retained civil servants and attract good quality staff into the system. 
- 12 core Ministries have been restructured (State House, Finance, Solid Minerals, National Planning Commission, FCT Administration, Health, Education, Foreign Affairs (pre-January 2007 part), Commerce (pre-January 2007 part), Science and Technology (pre-January 2007 part), Transport (pre-January 2007 part), and Internal Affairs (pre-January 2007 part)). The Government in January reduced the number of Ministries by merging some of them.

- $\quad$ Complete the restructuring of ministries, departments and agencies, (MDAs) and parastatals in 2nd Quarter, 2007. Progress has been made in this area; the reorganization of the CBN, NBS, and Center for Management Development has been completed, and the reorganizations of NNPC and FIRS are at an advanced stage. Specifically for the FIRS, the restructuring is focusing on the completion of the implementation of its human resource management system, including the installation of HRM hardware and software for nationwide access, and conducting a competence assessment exercise to determine training needs.

- $\quad$ The FIRS has made progress in the conduct of its nationwide taxpayer enumeration, preparatory to the introduction of an automated tax administration system, including TIN. A consultant was engaged for this purpose in late 2006 and is currently conducting the enumeration exercise. The project is now expected to be completed in July 2007.

- A Tax Policy Unit has been set up in the Ministry of Finance designed to help strengthen this aspect of the work of the fiscal authorities. Its capacity is being strengthened through internal reorganization of the personnel, and familiarizing it with the work of the FIRS so as to better define a niche for it and avoid possible duplication of roles.

- $\quad$ The National Bureau of Statistics (NBS) continues to be strengthened through its own internal reforms, with a view to repositioning it for more efficient delivery of macroeconomic statistics. It continues to work towards the introduction of quarterly GDP data, having completed a comprehensive economic survey in collaboration with the CBN.

\section{Transparency and anti-corruption efforts}

- The final result of the 1999-2004 audit of the oil and gas sector conducted under the auspices of the NEITI (a structural assessment criterion) was submitted to the Presidentin-Council in December 2006 and also shared with the public. ${ }^{5}$ The post-audit remedial measures were also approved by the Cabinet, and these are currently being put in place

\footnotetext{
${ }^{5}$ There was an unaccounted for monetary difference of about 3 percent in the original report published in Q1 2006, which the auditors indicated was within the margin of error of the profession. However, Mr. President instructed that further work be done to explain this difference, and this was fully explained (to 99.9 percent) in the final report.
} 
to address the areas of weakness identified in the reports. These include designing standard reporting templates for the relevant agencies and oil companies. Similarly, the Department for Petroleum Resources (DPR) is working with international partners, including Norway, on systems to improve the metering infrastructure in the upstream. The contract of the Hart Group (the auditors) has been extended through a 'change order' arrangement for the purpose of conducting the 2005 audit; the extension contract was signed in March, and work has commenced. The process of procuring an auditor for the 2006 and value-for-money audit was launched in April; tenders have been called for.

- The publication of the revenue allocation to the 3 tiers of Government is being continued.

- $\quad$ The EFCC has continued to make progress in its anti-corruption work, including in terms of arrest and prosecution of corrupt public officials and the screening of politicians vying for public office. In order to sustain the current status of Nigeria as having been de-listed from the category of non-cooperating countries and territories under the FATF, the EFCC and other agencies are intensifying their work in checking the activities of internet fraudsters and anti-money laundering. In a related move a nationwide corruption survey was conducted as part of the 2006 SEEDS benchmarking (a benchmark) with focus on public procurement and transparency and prosecution of corruption cases.

\section{The push to get relevant bills in the National Assembly passed has intensified in} recent months, as the Government strives to institutionalize the reforms, including:

- The passage of the Fiscal Responsibility Bill by the Senate took place in February. The Lower House has now also passed the bill.

- $\quad$ The Public Procurement Bill has been passed by both Houses of the National Assembly; including on the basis of another study tour in late 2006 by the relevant Committees.

- $\quad$ Six of the eight Tax Reform Bills have been passed by the National Assembly, and efforts are on to pass the other two; and

- $\quad$ Following more intensive engagement with the Senate, the NEITI bill was passed by the Senate in March; the House of Representatives had already passed its own version of the bill.

It is expected that harmonization will be concluded and all bills signed into law before the end of the current session.

11. The major challenge continues to revolve around how to transmit the gains of the reforms to everyday benefits for ordinary Nigerians as a means of securing their continued support for the reforms. In this context, the Government is continuing to lay emphasis on policies that will promote economic growth, particularly in the non-oil sector such 
as agriculture, solid minerals, and tourism. These would help towards achieving the MDG targets (as also captured under the NEEDS program) - poverty reduction, wealth creation, employment generation, etc. This will entail greater investment in physical and human infrastructure (education and health), productive activities, and a more vigorous pursuit of the Nigerian content agenda. In this context, the Government is targeting an annual real GDP growth rate of about 7-10 percent (it is estimated that a 7 percent growth rate is needed in order to achieve the objectives of NEEDS). Current estimates indicate that there is a financing gap of about $\$ 4$ billion per annum if Nigeria is to meet the NEEDS/MDG objectives. The development of the second phase of the NEEDS program (2008-2011) is at an advanced stage.

\section{Government continues to pursue its policy of deploying the savings arising from} exiting the Paris Club debt (about $\$ 1$ billion per annum, of which $\$ 750$ million is from the Federal Government) into MDG-related spending. In the 2007 budget, a total of N110 billion was appropriated for this purpose, following on the N100 billion allocated in 2006.

13. Program monitoring. The Government is continuing with the PSI arrangement involving quantitative targets, assessment criteria and structural benchmarks up to the first half of 2007 and in the context of the agreed framework (see tables 1 and 2). Targets will continue to be used to assess the performance of Government policy and reform implementation. We welcome the Fund's continued engagement with Nigeria to monitor the program performance under the IMF's Policy Support Instrument. In the light of the expected change of Administration, the Government envisages that the fourth and final review to assess the performance up to June 2007 will be no later than October 16, 2007.

\section{The Government reiterates its commitment to seek only concessional financing in}

the period ahead to help finance any gap in its investment program. However, an exceptional loan is being taken from China in the amount of $\$ 2.5$ billion towards the cost of building a railway system and other infrastructure projects for which a framework has been agreed. The terms of the loans are still under discussion, but the interest rate is likely to be about $3 \%$, with a grace period of 3-5 years and a term of 15-25 years depending on the particular project. The Government believes that this one-off arrangement is essential to meet its desire to strengthen the infrastructural base needed to promote economic development. It believes that the implementation of Nigeria's home-grown program supported under the PSI and its financing, as described above, will go a long way in helping the country achieve the MDGs. It further renews its commitment not to request for the use of IMF or PRGF resources in the course of implementation of its home-grown program. 


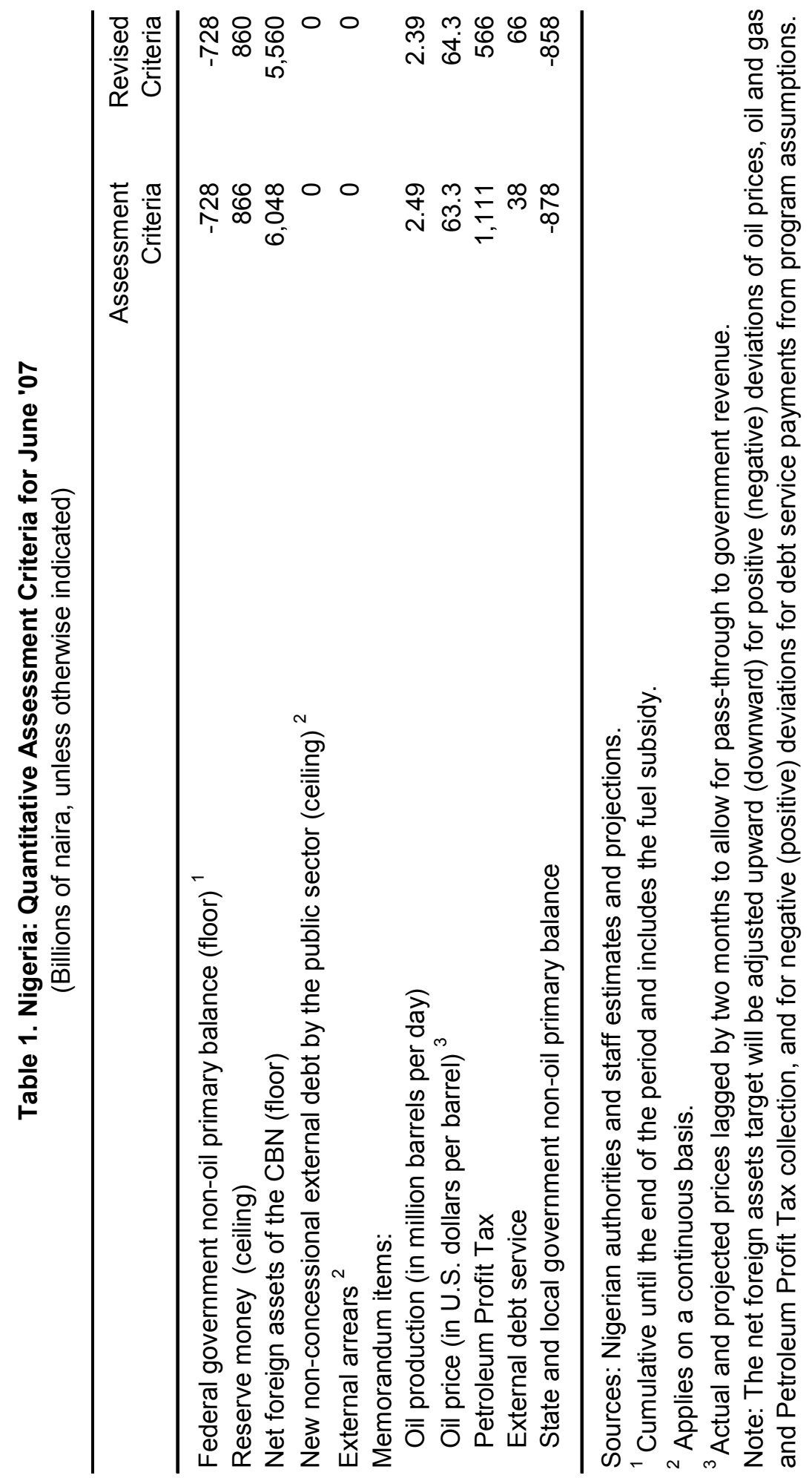


Table 2. Nigeria: Structural Benchmarks and Assessment Criteria for May-June 2007 under the PSI Program ${ }^{1}$

\begin{tabular}{|c|c|c|c|}
\hline $\mathrm{S} / \mathrm{N}$ & Structural Assessment Criteria & $\begin{array}{l}\text { Expected date } \\
\text { of achievement }\end{array}$ & Remarks \\
\hline 1 & $\begin{array}{l}\text { Bid opening for sale of the Abuja Electricity Distribution Plc as } \\
\text { set out in paragraph } 9 \text { of the statement }\end{array}$ & End June 2007 & $\begin{array}{l}\text { Ongoing; on } \\
\text { target }\end{array}$ \\
\hline 2 & $\begin{array}{l}\text { Issue report on SEEDS Benchmarking for } 36 \text { States to be } \\
\text { published as set out in paragraph } 9 \text { of the statement }\end{array}$ & End June 2007 & Ongoing \\
\hline 3 & $\begin{array}{l}\text { Complete restructuring of MDAs as set out in paragraph } 9 \text { of the } \\
\text { statement }\end{array}$ & End-May 2007 & Ongoing \\
\hline 4 & $\begin{array}{l}\text { FIRS to complete implementation of human resource management } \\
\text { system, including installation of HRM hardware and software for } \\
\text { nationwide access, and conduct a competence assessment exercise } \\
\text { to determine training needs. }\end{array}$ & End-June 2007 & Ongoing \\
\hline \multirow[t]{2}{*}{5} & $\begin{array}{l}\text { The Central Bank to introduce a new Standing Facility to help } \\
\text { reduce interest rate volatility as stated in paragraph } 7 \text { of the } \\
\text { statement. }\end{array}$ & End-June 2007 & Done \\
\hline & Structural Benchmarks & & \\
\hline 1 & $\begin{array}{l}\text { Continue publication of revenue allocation to the three tiers of } \\
\text { Government }\end{array}$ & Continuous & Ongoing \\
\hline 2 & $\begin{array}{l}\text { Complete restructuring of five parastatals in terms of right-sizing } \\
\text { and right-staffing. }\end{array}$ & End-May 2007 & Ongoing \\
\hline 3 & Bid opening for sale of 8 Oil Service Companies & End-June 2007 & Done \\
\hline 4 & $\begin{array}{l}\text { Nigerian Customs Service to conduct staff survey to determine } \\
\text { suitability of personnel for minimum requirements of service in } \\
\text { preparation for rationalization, realignment and right-sizing its } \\
\text { human resource system. }\end{array}$ & End-June 2007 & Done \\
\hline
\end{tabular}

\footnotetext{
${ }^{1}$ Assessment criteria will also apply on a continuous basis to exchange and import measures as in Fund supported programs.
} 


\section{Attachment II. Nigeria: Technical Memorandum of Understanding (TMU)}

May 2007

1. This Memorandum of Understanding between the Nigerian authorities and the IMF staff sets out the definitions of performance targets, as well as reporting requirements for the Nigerian reform program supported under the Policy Support Instrument (PSI). The performance targets are reported in Table 1 of the authorities' policy statement dated May 2007.

\section{Quantitative Performance Targets: Definitions and Reporting Standards}

\section{A. Floor on Federal Government Non-Oil Primary Balance}

2. Definition: The non-oil primary balance of the federal government will be measured on a cash basis and will be defined as non-oil-and-gas-related revenue minus total expenditure excluding interest payments. Non-oil-and-gas-related revenue consists of the federal government's share of imports and excise duties, companies' income tax, and valueadded tax, as well as the federal government's independent revenue. Federal government expenditure includes recurrent and capital expenditure, as well as any clearance of expenditure arrears and recapitalizations. Capital expenditure on a cash basis is defined as the utilization of capital releases from the current and previous budgets during the relevant period. Interest payments consist of domestic and external interest payments. The non-oil primary balance target is defined as non-oil revenue received less non-interest expenditures incurred from the beginning of the year.

3. Supporting material: Data on federally collected revenue and federal expenditure will be provided by the Office of the Accountant General of the Federation (OAGF) to the Fund within six weeks of the end of each month. The OAGF will also provide to the Fund monthly federal government capital account balances within six weeks of the end of each month, and a quarterly summary of capital releases and utilization (for each budget year) within six weeks of the end of each quarter.

\section{B. Ceiling on Reserve Money}

4. Definition: Reserve money is defined as currency in circulation and deposit money banks' deposits at the Central Bank of Nigeria (CBN). The reserve money target is defined as the end-of-period stock.

5. Supporting material: The CBN balance sheet is to be transmitted to the Fund on a monthly basis within six weeks of the end of each month. 


\section{Floor on Net Foreign Assets of the CBN}

6. Definition: Net Foreign Assets (NFA) of the CBN are defined as foreign assets minus short-term foreign liabilities of the CBN. The NFA target is defined as the end-ofperiod stock.

7. Adjustment clauses: The floor will be adjusted to reflect cumulative deviations from program assumptions on (a) oil revenue and (b) external debt service payments. The floor will be adjusted upward (downward) to the extent that receipts of oil revenue into the federation account of the CBN exceed (fall short of) the programmed levels on account of higher (lower) than programmed oil and gas prices and production volumes, and upward (downward) to the extent that petroleum profit tax collections exceed (fall short of) programmed levels. The floor will be adjusted upward (downward) to the extent that external debt service payments fall short of (exceed) the programmed level. Programmed levels of oil prices and volumes and of external debt payments are specified in Table 1 of the authorities' policy statement.

8. Supporting material: Data on NFA, foreign assets, foreign liabilities will be provided by the CBN and data on oil revenue into the federation account of the CBN and on petroleum profit tax collection will be provided by the OAGF to the Fund on a monthly basis within six weeks of the end of each month. Data on external debt service payments (principal, interest, and total) broken down by creditor will be supplied by the Debt Management Office (DMO) to the Fund on a monthly basis within four weeks of the end of each month.

\section{Non-Accumulation of External Arrears}

9. Definition: During the period of the PSI, the federal government and the CBN will not incur any payment arrears on external debt service obligations to creditors. Official external payment arrears are defined as unpaid debt service by the federal government and the CBN beyond the due date. This definition excludes arrears subject to future rescheduling according to agreements with the Paris Club or bilateral creditors. The assessment target on non-accumulation of external arrears is continuous.

10. Supporting material: Details of arrears accumulated on interest and principal payments to creditors will be reported by the DMO to the Fund within two weeks from the due date of the missed payment 


\section{E. Ceiling on Contracting or Guaranteeing of New Non-Concessional External Debt by the Federal Government and the CBN}

11. Definition: Non-concessional external loans are defined as loans from lenders other than the IMF with a grant element of less than 35 percent of the value of the loan. The grant element is to be calculated by using currency-specific discount rates reported by the OECD (CIRRs). ${ }^{1}$ For maturities of less than 15 years, the grant element will be calculated based on six-month averages of commercial interest rates. For maturities longer than 15 years, the grant element will be calculated based on 10-year averages. This assessment criterion applies to debt and to commitments contracted or guaranteed for which value has not been received. ${ }^{2}$ Previously contracted non-concessional external debt that has been rescheduled will be excluded from the definition of "new debt" for the purposes of this assessment criterion.

12. Supporting material: Details of all new commitments and government guarantees for external borrowing, with detailed explanations, will be provided by the Ministry of Finance to the Fund on a monthly basis within two weeks of the end of each month.

\section{Memorandum Item: Definitions and Reporting Standards}

\section{F. Floor on Non-oil Primary Balance of States and Local Governments (SLGs)}

13. Definition: The non-oil primary balance of the SLGs will be measured as the non-oiland-gas-related revenue minus primary expenditure. Non-oil-and-gas-related revenue consists of the SLGs' share of imports and excise duties, companies' income tax, and valueadded tax. SLGs primary expenditure will be computed as (i) the non-oil-and-gas-related revenue; plus (ii) the oil-and-gas-related revenue including derivation and the distribution of accumulated excess crude proceeds; plus (iii) change in net claims on SLGs by the banking system (excluding changes in excess crude deposits); minus (iv) external debt service on a cash basis.

14. Supporting material: Data on SLGs revenue will be provided by the Office of the Accountant General of the Federation (OAGF) to the Fund within six weeks of the end of each month. The CBN will provide data on changes in net claims (excluding changes in excess crude deposits) on SLGs by the banking system.

\footnotetext{
${ }^{1}$ An electronic spreadsheet file that shows the relevant discount rates reported by the OCD (CIRRs) will be provided on a periodic basis by Fund staff.

${ }^{2}$ For this purpose, debt is defined as in IMF guidelines on external debt.
} 


\section{ANNEX I. RELATIONS WITH THE FUND}

(As of April 30, 2007)

I. Membership Status: Joined: 03/30/1961; Article XIV

II. General Resources Account:

Quota

Fund Holdings of Currency

Reserve position in the Fund

III. SDR Department:

Net cumulative allocation

Holdings
SDR Million

$1,753.20$

1,753.11

0.14

SDR Million

157.16

1.97
\%Quota

100.00

100.00

0.01

\%Allocation

100.00

1.25

IV. Outstanding Purchases and Loans: None

V. Latest Financial Arrangements

$\begin{array}{lllcc}\text { Type } & \text { Approval Date } & \text { Expiration Date } & \begin{array}{c}\text { Amount } \\ \text { Approved } \\ \text { (SDR million) }\end{array} & \begin{array}{c}\text { Amount } \\ \text { Drawn } \\ \text { (SDR million) }\end{array} \\ \text { Stand-by } & \text { Aug. 04, 2000 } & \text { Oct. 31, 2001 } & 788.94 & 0.00 \\ \text { Stand-by } & \text { Jan. 09, 1991 } & \text { Apr. 08, 1992 } & 319.00 & 0.00 \\ \text { Stand-by } & \text { Feb. 03, 1989 } & \text { Apr. 30, 1990 } & 475.00 & 0.00\end{array}$

\section{Projected Obligations to Fund}

(SDR Million; based on existing use of resources and present holdings of SDRs):

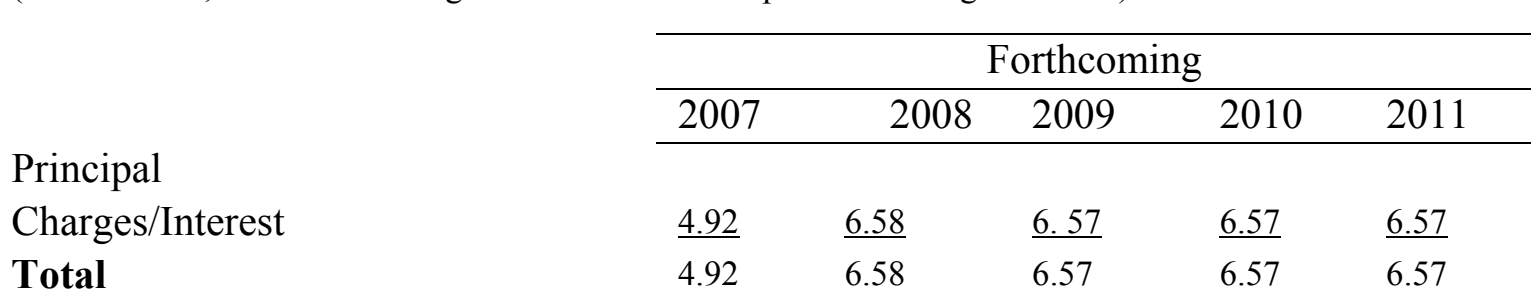

\section{Exchange Rate Arrangement:}

25. Nigeria's current exchange rate arrangement is de jure a managed float with no preannounced target for the exchange rate of the naira. On February 20, 2006, the CBN executed its first auction under the new wholesale Dutch Auction System (DAS). The introduction of the wholesale DAS with free sale to the interbank market was an important step towards unification of the two major foreign exchange markets - the retail market for nonfinancial traders and investors and the interbank market. The naira also continues to be traded in two markets for small-scale cash transactions - the bureau de change market and the curb market. Since May 2006 the exchange has effectively been unified. The introduction 
of the wholesale DAS also represents an important step toward accepting the obligations of Article VIII, sections 2, 3 and 4 of the IMF's Articles of Agreement, although the new system may still entail a multiple currency practice. A comprehensive assessment by MCM and LEG is now needed to identify the extent of remaining restrictions and multiple currency practices.

\section{Safeguards Assessment}

Under the Fund's safeguards assessment policy, the Central Bank of Nigeria (CBN) was subject to a full safeguards assessment with respect to the Stand-By Arrangement, which expired on October 31, 2001. The assessment, which included an on-site visit, was completed on November 28, 2001. The assessment concluded that vulnerabilities existed in the areas of financial reporting and legal structure of the Central Bank. Staff findings and proposed recommendations are reported in the Country Report No. 03/3.

\section{Article IV Consultation}

Executive Board endorsement of Nigeria's request for a Policy Support Instrument automatically placed Nigeria on the 24-month Article IV consultation cycle. The last Article IV consultation discussions were held in Lagos and Abuja during the period March 8-25, 2005. The staff report (Country Report No. 05/302) was discussed by the Executive Board and the consultation concluded on July 18, 2005.

\section{Technical Assistance (TA) Since 2000:}

\section{Department Purpose of TA mission}

FAD Fiscal regime for oil and gas

STA Balance of payments statistics

FAD Resident treasury advisor

FAD Resident budget advisor

FAD Fiscal federalism

STA Government finance statistics

FAD Expenditure management

STA Money and banking statistics

MAE Foreign exchange management

FAD Resident budget advisor

MAE FSAP

MAE FSAP

FAD Public expenditure management

STA Government finance statistics

\section{Duration}

February 8-26, 2000

November 8-21, 2000

November 25, 2000-February 25-2001

and April 8-July 7, 2001

January 11-March 11, 2001

January 24-Feb. 11, 2001

January 21-Feb. 3, 2001

February 2-10, 2001

March 22-April 4, 2001

May 28-June 8, 2001

October 2000-Dec. 2001

December 5-15, 2001

February 4-20, 2002

January 29-February 8, 2002

February 28-March 13, 2002 


\begin{tabular}{|c|c|c|}
\hline FAD & Resident budget advisor & April 19 2002-June, 2003 \\
\hline MFD & Domestic debt management & February 25-March 5, 2003 \\
\hline STA & General data dissemination standards & July 2-15, 2003 \\
\hline STA & National accounts & July 24-August 12, 2003 \\
\hline FAD & Budget process reforms & August 20-29, 2003 \\
\hline FAD & Pension reform & October 20-29, 2003 \\
\hline FAD & Public expenditure management advisor & February-August 2004 \\
\hline MFD & Domestic debt management & February 5-17, 2004 \\
\hline LEG & FIU creation and organization & May 31-June 04, 2004 \\
\hline LEG & Legislative drafting/FIU & July 12-16, 2004 \\
\hline FAD & Tax administration & July 19-August 3, 2004 \\
\hline MFD & Monetary operations/foreign exchange & August 26-September 10, 2004 \\
\hline FAD & Public expenditure management advisor & October 2004-June 2005 \\
\hline LEG & Exchange rates systems & November 16-22, 2004 \\
\hline MFD & Bank supervision/restructuring & November 16-29, 2004 \\
\hline STA & Balance of payment statistics & February 2-16, 2005 \\
\hline FAD & Tax administration & February 8-21, 2005 \\
\hline MFD & Banking supervision, financial, exchange & \\
\hline \multirow[b]{2}{*}{ MFD } & Market, and currency reforms & March 9-24, 2005 \\
\hline & $\begin{array}{l}\text { Banking consolidation and supervision, } \\
\text { Currency reforms }\end{array}$ & August 29-September 13, 2005 \\
\hline FAD & Tax administration peripatetic advisor & July -December, 2005 (3 visits) \\
\hline FAD & Public expenditure management advisor & October 2005-April 2006 \\
\hline MFD & Banking consolidation, monetary policy & November 9-22, 2005 \\
\hline MFD & Monetary operations advisor & December $11-17,2005$ \\
\hline STA & Money and banking statistics & January 26-February 8, 2006 \\
\hline MFD & Exchange rate management advisor & February 13-20, 2006 \\
\hline MFD & Banking consolidation, monetary policy & February 27-March 10, 2006 \\
\hline STA & Balance of payment statistics & March 15-28, 2006 \\
\hline $\mathrm{MCM}$ & Monetary policy peripatetic advisor & $\begin{array}{l}\text { Since May } 20064 \text { visits (some joint } \\
\text { with HQ staff) }\end{array}$ \\
\hline MCM & Financial sector development & November 17-24, 2006 \\
\hline MCM & Monetary policy long term advisor & since December 18, 2006 \\
\hline MCM & Central bank accounting & January 28-February 28, 2007 \\
\hline FAD & Public financial management & February 14-23, 2007 \\
\hline STA & Monetary statistics & February 20-27, 2007 \\
\hline
\end{tabular}

\section{Resident Representative:}

Mr. Michael Bell entered on duty as Senior Resident Representative in Abuja on August 10, 2006. 


\section{ANNEX II. RELATIONS WITH THE WORLD BANK}

\section{Partnership for development}

1. The Nigerian government has been making progress with implementation of the National Economic Empowerment and Development Strategy (NEEDS), a program of reforms aimed at achieving economic and social transformation in Nigeria. This effort at the Federal level, working in parallel with State Economic Empowerment and Development Strategies (SEEDS) at the state level, is continuing to improve the environment for development assistance and strengthen the framework for aid effectiveness.

2. The World Bank assistance to Nigeria is based on a joint World Bank/DFID Country Partnership Strategy (CPS) prepared in consultation with government, civil society, private sector and other development partners and approved by the British Government and the Board of the World Bank on June 25, 2005. This strategy, which will guide the assistance of the Bank and UK's DFID to Nigeria for the period FY06 to FY09, supports selected priorities under the three broad pillars of the NEEDS/SEEDS framework, namely: (i) empowering people through improved service delivery for human development; (ii) promoting private enterprise through improved business environment for non-oil growth and improved access to productive infrastructure; and (iii) changing the way government does its work through improved transparency and accountability.

3. Under the CPS, both the World Bank and UK's DFID are deepening engagement with Nigeria, in response to the improved policy environment and the importance of Nigeria for meeting the MDGs in Africa. More support is provided to states where constitutional responsibility for most functions related to achievement of the MDGs lies. At the same time, the Bank and DFID are more selective in approach, concentrating assistance at the Federal level and in a set of lead states that are strongly committed to reforms and where development assistance can achieve the greatest impact on growth and poverty reduction. At the moment, the bank has signed State Partnership Agreements with four of such lead states.

4. Under the strategy the IDA lending annual envelope has been increased from about US\$500 million to about US\$750 million for FY07-10. This envelope is expected to increase as Nigeria's policy performance continues to improve. Indicative lending plans for the remaining two years of the CPS will be set during the mid-term review of the CPS in 2007.

\section{World Bank-IMF relations}

5. The IMF and World Bank staffs maintain a close collaborative relationship on Nigeria. Both institutions are coordinating their policy advice to the government in several areas (including the financial sector, public financial management, and statistics) through collaboration on analytical work, and through joint technical assistance missions. The Bank staff is regularly invited to join IMF macroeconomic missions. The Bank's work and support to Government in key structural reform areas informs the PSI program. 
6. In 2005, the Bank and the IMF staff collaborated on a Debt Sustainability Analysis for Nigeria and prepared a Joint Staff Assessment Note on the NEEDS which was discussed by both the Boards of the World Bank and the IMF. In 2006, the Bank and the IMF staff jointly updated the Debt Sustainability Analysis.

7. Bank and IMF staffs are beginning work with the Government on the macroeconomics of scaling up. This exercise will analyze options for scaling up to meet the MDGs in Nigeria in a manner consistent with macroeconomic stability objectives.

\section{Lending activities}

8. The IDA commitment in Nigeria continued to show an upward trend; growing from about US\$1.5 billion in FY05, US\$1.8 billion in FY06 and currently (FY07) stands at about US\$2.3 billion; out of which only about 18 percent is at risk. This represents a significant improvement compared to 79 percent in FY03 and 43 percent in FY05 respectively. This portfolio comprises 21 projects covering activities in various sectors of the economy including Health and Social Services (25 percent), Education (8 percent), Transportation (6 percent), Energy and Mining (14 percent), Public Administration/Law (18 percent), Agriculture (4 percent), Water Supply/Sanitation/Flood Protection (23 percent), Finance (1 percent), Industry and Trade (1 percent).

9. As of April 2007, about US\$842 million has been disbursed out of the total IDA commitment of US $\$ 2.3$ billion. With the current disbursement ratio of 19.4 percent, the portfolio's level of efficiency in the resource utilization is likely to meet or surpass the 23 percent level achieved at the end of FY06. The recent improvement in disbursement could be explained by both the intensification of project supervision efforts and the maturity of the project portfolio (with the average project age of over three years). The Bank continues to work towards mitigating the risks faced by the portfolio and focuses on strengthening monitoring and evaluation (M\&E) arrangements to ensure that projects in the portfolio achieve their development objectives.

\section{Non-lending activities}

10. The World Bank has continued to expand its program of economic and sector analysis as part of its efforts to strengthen policy dialogue and improve on the quality of its investment operations. The current Economic and Sector Work program has a strong focus on public expenditure management and competitiveness and growth. It is also has been paying growing attention to regional integration agenda, poverty analysis, and trends in the social sector. Key proposed analytical pieces include a Fiscal Federalism study (FY08), an Agriculture Financing Review (FY08), an Agriculture PER (FY08), an Employment and Growth study (FY09), a Poverty Assessment (FY10) and a Trade and Regional Integration study (FY10). 


\section{International Finance Corporation (IFC) activities}

11. Nigeria remains IFC's largest commitment portfolio in Sub-Sahara Africa with an outstanding portfolio of US\$612 million in private sector investments as of March 2007. IFC's portfolio in the country represents approximately 26 percent of the Sub-Sahara Africa region's portfolio, having grown from US\$143 million in 2001. While IFC continues to make direct investments in projects that will assist Nigeria diversify its economy from the oil sector, IFC has worked with other development partners to support growth of Micro, Small and Medium Enterprises (MSMEs) through a range of technical assistance initiatives. Highlights of IFC activities in the various sectors of Nigeria are described below.

12. Financial sector. Over the past five years, IFC has disbursed over US\$220 million to five Nigerian banks in terms of long-term credit lines. It also signed on five Nigerian banks as issuing banks under the IFC Global Trade Finance Program. The total utilization of the trade finance facilities was approximately US\$200 million in FY07. In addition, IFC is providing Nigerian banks with specialized financing facilities and technical assistance, to open up financing opportunities in new or untapped markets such as women entrepreneurs and construction finance. IFC's involvement has helped its portfolio banks strengthen their governance and risk management capabilities. Moreover, IFC is increasingly diversifying to finance non-bank financial institutions such as insurance and asset management companies. IFC has been able to provide naira long-term financing to both banks and real sector companies because of the existence of long-term cross-currency swaps from naira into convertible currencies.

13. General manufacturing services. Over the years, IFC under the African Enterprise Funds has invested in a variety of general manufacturing projects, and it recently disbursed part of a long-term loan of US\$75 million for the Obajana Cement Company. This is a green field cement plant with an annual capacity of 4.4 million tons. The project also includes a $135 \mathrm{MW}$ power plant, a $92 \mathrm{KM}$ natural gas pipeline, a water supply dam and other support facilities.

14. Oil and gas, mining and petrochemicals. IFC's strategy in this sector has been to target the services area in linkages to projects with the oil majors, as well as indigenous oil and gas production and services companies. There have also been recent opportunities in the petrochemicals sector as a result of privatizations, such as the Eleme petrochemicals project, where IFC has approved a US\$75 million investment for rehabilitation and expansion of capacity.

15. Advisory and technical assistance projects. Private Enterprise Partnership (PEP) Africa initiative designs and implements programs to support the development of the private sector in three areas: improving the investment climate, proactively identifying investment opportunities, and supporting SME development. In Nigeria, under PEP Africa, the leading projects include the SME Entrepreneurship Development Initiative and the Oil Sector SME Linkages Project in coordination with INSOK from Norway, and bond market development. 
IFC is also the lead adviser to the government for the privatization of Nigeria airports, beginning with the Abuja airport. In the Power sector, a joint World Bank/IFC Energy Team is working with the federal government on sector reform and strategies for privatization.

\section{World Bank Institute activities (WBI)}

16. Nigeria is a WBI Focus Country. WBI has continued to partner with relevant stakeholders in building capacity for the implementation of programs that will empower communities, improve economic governance and ensure non-oil private sector development. WBI also aims to strengthen the national parliament, increase civil society participation in governance through youth programs, facilitate the development of good quality and pluralistic media, and support science and technology developments by assisting the African Institute of Science and Technology (AIST). A Global Distance Learning Network (GDLN) center is expected to be completed and functional in Abuja before mid-2007. WBI cooperates with the Federal Center for Health Systems Studies through its training programs of key managers and decision makers involved in health sector reforms. Other important WBI programs include: Support for NEEDS Implementation, HIV/AIDS, and building capacity in Nigerian youth organizations.

\section{Multilateral Investment Guarantee Agency (MIGA)}

17. MIGA has continued to increase its involvement in the promotion of foreign direct investment in Nigeria by offering guarantees against noncommercial risks to foreign investors. MIGA has issued guarantees to investors in various sectors of the Nigerian economy totaling about US\$91.5 million in gross exposure (1.88\% of the Agency's gross portfolio). The major stakes of MIGA in Nigeria has been in the services sector, where MIGA issued guarantees for two separate companies (SGS and Cotecna) for a total amount of US\$81 million in FY06. The objective of the two projects is to modernize the ports screening facilities in order to reduce both the time required for customs clearance and opportunities for graft. The Agency has also played an effective role in resolving a potential investment dispute between the Government of Nigeria and one insured investor. 
Table 1. Nigeria: IDA Credit Portfolio

(As of April 23, 2007, US\$ million)

\begin{tabular}{|c|c|c|c|c|c|}
\hline $\begin{array}{l}\text { Project } \\
\text { ID }\end{array}$ & Project Name & $\begin{array}{l}\text { Fiscal } \\
\text { Year }\end{array}$ & $\begin{array}{l}\text { Approved } \\
\text { IDA } \\
\text { Amount }\end{array}$ & Disbursed & Undisbursed \\
\hline & & & & & 29.1 \\
\hline & Economic Management Capacity Building & & & & 1.5 \\
\hline P069892 & $\begin{array}{l}\text { Local Empowerment \& Environment } \\
\text { Management }\end{array}$ & 2004 & 70.0 & 43.0 & 36.0 \\
\hline P069901 & Community-based Urban Development & 2002 & 110.0 & 34.9 & 97.6 \\
\hline P070290 & 2nd Health Systems Development & 2002 & 127.0 & 115.2 & 36.2 \\
\hline P070291 & HIV/AIDS Program Development & 2002 & 90.3 & 86.6 & 18.4 \\
\hline P070293 & Privatization Support Project & 2001 & 114.3 & 63.4 & 70.6 \\
\hline P071075 & Urban Water Sector Reform I & 2004 & 120.0 & 18.3 & 104.9 \\
\hline P072018 & Transmission Development & 2002 & 100.0 & 82.9 & 33.2 \\
\hline P074963 & Lagos Urban Transport Project & 2003 & 150.0 & 76.5 & 86.7 \\
\hline P080295 & Polio Eradication & 2003 & 80.4 & 75.7 & 3.3 \\
\hline P083082 & Micro, Small \& Medium Enterp. & 2004 & 32.0 & 4.4 & 29.5 \\
\hline P086716 & $\begin{array}{l}\text { Sustainable Management of Mineral } \\
\text { Resources }\end{array}$ & 2005 & 120.0 & 17.2 & 102.9 \\
\hline P088150 & Economic Reform and Governance & 2005 & 140.0 & 25.0 & 116.2 \\
\hline P071391 & Urban Water Sector Reform II & 2006 & 200.0 & 11.8 & 188.9 \\
\hline P074447 & $\begin{array}{l}\text { State Governance and Capacity Building } \\
\text { Project }\end{array}$ & 2005 & 18.1 & 1.0 & 16.9 \\
\hline P090104 & National Energy Development Project & 2006 & 172.0 & 5.4 & 166.7 \\
\hline P100122 & Avian Influenza Control & 2006 & 50.0 & 20.4 & 32.2 \\
\hline P071340 & $\begin{array}{l}\text { Lagos Metropolitan Development and } \\
\text { Governance }\end{array}$ & 2007 & 200.0 & 0.0 & 210.2 \\
\hline P102966 & $\begin{array}{l}\text { Community-based Poverty Reduction } \\
\text { Project }\end{array}$ & 2000 & 85.0 & 68.0 & 25.1 \\
\hline \multirow[t]{2}{*}{ P097921 } & Malaria Control Booster Project & 2006 & 180.0 & 0.0 & 184.2 \\
\hline & TOTAL & & $2,279.1$ & 842.5 & $1,590.4$ \\
\hline
\end{tabular}


Table 2. Nigeria: Proposed Lending Summary, FY2008/10

(As of April 23, 2007, US\$ million)

\begin{tabular}{|c|c|c|}
\hline \multirow{7}{*}{ FY 08} & Fadama 3 & 200 \\
\hline & Federal Roads & 250 \\
\hline & State Education & 50 \\
\hline & Rural Access and Mobility (RAMP 1) & 70 \\
\hline & $\begin{array}{l}\text { Commercial Agriculture } \\
\text { State Level Growth Pole \& Infrastructure Facilities }\end{array}$ & 100 \\
\hline & & 80 \\
\hline & Total & 750 \\
\hline \multirow[t]{8}{*}{ FY 09} & State Health or Polio Immunization Project & 50 \\
\hline & $\begin{array}{l}\text { Support for Sustainable development of Power } \\
\text { Sector }\end{array}$ & 80 \\
\hline & Federal Roads & 250 \\
\hline & State and Local Government/Justice project & 50 \\
\hline & Community Social Development project & 200 \\
\hline & HIVIAIDS & 100 \\
\hline & Lagos-Abidjan Transport and Transit (Regional) & 20 \\
\hline & Total & 750 \\
\hline \multirow[t]{9}{*}{ FY 10} & Commercial Agriculture/Rural Finance & 100 \\
\hline & Federal Roads 2 & 250 \\
\hline & State Education 3 & 50 \\
\hline & State Water & 100 \\
\hline & State Health & 50 \\
\hline & Rural Access and Mobility (RAMP 2) & 150 \\
\hline & $\begin{array}{l}\text { Conditional Cash Transfer /Maternal and Child } \\
\text { Health }\end{array}$ & 100 \\
\hline & Total & 750 \\
\hline & & \\
\hline
\end{tabular}


Table 3. Nigeria: Non-lending Summary, FY2008/10

(As of April 23, 2007)

\begin{tabular}{|c|c|}
\hline \multicolumn{2}{|l|}{ Proposed } \\
\hline \multirow[t]{6}{*}{ FY08 } & $\begin{array}{l}\text { Polio Eradication ICR } \\
\text { Economic Management and Capacity-building Project (EMCAP) ICR }\end{array}$ \\
\hline & Agriculture Public Expenditure Review \\
\hline & Fiscal Federalism Study \\
\hline & Niger Delta Social and Conflict Analysis \\
\hline & $\begin{array}{l}\text { Financing Agriculture Study } \\
\text { Human Development Strategy Note }\end{array}$ \\
\hline & $\begin{array}{l}\text { Health Insurance Study } \\
\text { Petroleum Pricing Study } \\
\text { NEITI Report }\end{array}$ \\
\hline FY 09 & $\begin{array}{l}\text { Governance of Service Delivery Review } \\
\text { Employment and Growth Study } \\
\text { Transport and Economic Growth Study } \\
\text { Nutrition Study } \\
\text { Access to Justice study }\end{array}$ \\
\hline FY 10 & $\begin{array}{l}\text { Poverty Assessment } \\
\text { Trade and Regional Integration Study } \\
\text { Non-Bank Financial Institutions Study }\end{array}$ \\
\hline \multicolumn{2}{|l|}{ Ongoing } \\
\hline \multirow[t]{17}{*}{ FY $07-08-09$} & $\begin{array}{l}\text { Education Sector Review - States } \\
\text { Health Sector review - States }\end{array}$ \\
\hline & Investment Climate Assessment - State \\
\hline & Corruption and Governance Risks Assessment \\
\hline & Natural Resources Management/Environmental Policy Dialogue \\
\hline & Nigeria Extractive Industry Transparency Initiative (NEITI) \\
\hline & Legal reforms-Technical Assistance (TA) \\
\hline & $\begin{array}{l}\text { Niger Delta Community Foundation } \\
\text { Results Monitoring IDF }\end{array}$ \\
\hline & $\begin{array}{l}\text { Country Portfolio Performance Review (CPPR) } \\
\text { Implementation support-M\&E and PFMU }\end{array}$ \\
\hline & Human Development Outcome Dialogue \\
\hline & Non-oil Growth Outcome Dialogue \\
\hline & Governance Outcome Dialogue \\
\hline & Donor Harmonization \\
\hline & Economic Monitoring \\
\hline & Support to the National Assembly \\
\hline & Civil Society Outreach \\
\hline & Private Sector Outreach \\
\hline & Communication Outreach and Academia \\
\hline
\end{tabular}


Table 4. Nigeria: International Finance Corporation Statement of IFC's Held and Disbursed Portfolio at March 31, 2007

\begin{tabular}{|c|c|c|c|c|c|c|c|}
\hline Nigeria & Sector & $\begin{array}{c}\text { Fiscal Year in } \\
\text { which } \\
\text { commitments } \\
\text { were made }\end{array}$ & $\begin{array}{l}\text { Total } \\
\text { IFC }\end{array}$ & Loan & Equity & $\begin{array}{l}\text { Quasi- } \\
\text { loan/Quasi } \\
\text { equity }\end{array}$ & Guarantee \\
\hline & & & \multicolumn{5}{|c|}{ US\$ millions } \\
\hline AEF Global Fabri & Textiles & FY99 & 0.3 & 0.3 & - & - & - \\
\hline AEF Hercules & Education services & FY99 & 1.3 & 1.3 & - & - & - \\
\hline AEF Safety Center & Education services & FY00 & 0.37 & 0.37 & 0.0 & - & - \\
\hline AEF Vinfesen & Plastics \& Rubber & FY95 & 1.0 & - & - & 1.0 & - \\
\hline Abuja Intl & Health Care & FY94 & 1.75 & 1.75 & 0.0 & - & - \\
\hline Accion Nigeria & Finance \& Insurance & FY05 & 1.89 & - & 1.89 & - & - \\
\hline Adamac & Oil, Gas \& Mining & FY03 & 11.56 & 11.56 & - & - & - \\
\hline CAPE FUND & Finance \& Insurance & FY00 & 0.41 & - & 0.41 & & - \\
\hline Diamond Bank & Finance \& Insurance & FY00,05,06 & 50.0 & - & - & 50.0 & - \\
\hline FSB & Finance \& Insurance & FY00 & 5.4 & 3.15 & - & 2.25 & - \\
\hline $\mathrm{FSDH}$ & Finance \& Insurance & FY92,93 & 0.86 & & 0.86 & - & - \\
\hline GTB & Finance \& Insurance & FY00,04,05,06 & 74.0 & 74.0 & - & - & - \\
\hline GTFP Access Bank & Finance \& Insurance & FY06,07 & 44.13 & - & - & 15.0 & 29.13 \\
\hline GTFP Diamond Bank & Finance \& Insurance & FY06,07 & 26.12 & - & - & - & 26.12 \\
\hline GTFP GTB Nigeria & Finance \& Insurance & FY06,07 & 9.45 & - & - & - & 9.45 \\
\hline GTFP IBTC PIc. & Finance \& Insurance & FY06,07 & 1.51 & - & - & - & 1.51 \\
\hline GTFP Zenith & Finance \& Insurance & FY06,07 & 12.84 & - & - & - & 12.84 \\
\hline IBTC & Finance \& Insurance & FY00,06 & 50.0 & 20.0 & 15.43 & 14.57 & - \\
\hline Ikeja Hotel & Accommodation \& Tourism & FY81,85,88 & 0.1 & - & 0.1 & - & - \\
\hline MTNN & Information \& Technology & FY02,05 & 69.25 & 52.50 & 16.75 & - & - \\
\hline NTEF & Finance \& Insurance & FY02 & 20.0 & - & - & - & 20.0 \\
\hline Obajana Cement & Construction \& Real Estate & FY05 & 75.0 & 75.0 & - & - & - \\
\hline SOCKETWORKS & Education services & FY06 & 2.36 & - & - & 2.36 & - \\
\hline UPDC Hotels Ltd. & Accommodation \& Tourism & FY04 & 10.6 & 10.6 & - & - & - \\
\hline UBA & Finance \& Insurance & $\mathrm{FY} 02,04,07$ & 50.0 & - & - & 50.0 & - \\
\hline Hygea & Accommodation \& Tourism & FY04 & 10.6 & 10.6 & - & - & - \\
\hline LEADWAY & Education services & FY07 & 3.04 & 3.04 & - & - & - \\
\hline ELEME & Oil, Gas \& Mining & FY07 & 75.00 & 60.0 & - & 15.0 & - \\
\hline & & Total & 611.54 & 313.62 & 48.71 & 150.18 & 99.05 \\
\hline
\end{tabular}




\section{ANNEX III. STATISTICAL ISSUES}

1. Nigeria's statistical base continues to suffer from serious deficiencies that hamper surveillance, policy design, and monitoring. These deficiencies are across the board, but particularly affect the national accounts, government finance, money and banking, and the external accounts, including major inconsistencies between balance of payments and customs data on trade. Numerous problems prevent the compilation of timely and internally consistent data, in particular lack of data sharing between data producing and collecting agencies, and insufficient computerization. In addition, the 1957 Statistical Act no longer addresses the requirements of data collection and dissemination within a federal structure. The authorities recognize these problems, and intend to address them. In April 2003, Nigeria began participation in the Fund's General Data Dissemination System (GDDS), and posted its metadata on the Fund's dissemination standards bulletin board (DSBB). These metadata were subsequently updated towards the end of 2005 and the beginning of 2006.

\section{National accounts}

2. The national accounts statistics are of poor quality, largely owing to a deterioration in business and household surveys. Estimates of value added for agriculture, mining (oil), industry, transportation, and financial and other services are based on old surveys with extrapolations that use out-of-date ratios and other indicators. Many sectoral deflators are not soundly based and have a number of inconsistencies. In the past, the use of the official exchange rate has resulted in a gross understatement of value added in the oil and export sectors. The expenditure accounts suffer from unreliable external trade and government budgetary data. Annual national accounts statistics are published by the National Bureau of Statistics (NBS) with about a one-year lag.

3. A July 2003 mission to help the authorities implement the GDDS recommendations in the area of national accounts and provide guidance on institutional reforms in the statistical system, found that progress has been made and that the authorities were receiving significant technical assistance from DfID and other institutions such as the World Bank. Results of a 2003 household living standards survey are now available. The base year was updated to 1990 from 1984 to better reflect the economic structure of the economy. Moreover, the GDP methodology has been amended for estimates from 2004 onward in a move toward SNA 1993 and to incorporate new data sources. These revisions are currently being reviewed in the context of the IMF/ DFID GDDS Phase 2 Project for Anglophone Africa.

\section{Prices}

4. The official monthly consumer price index (CPI) is a composite of urban and rural price data, and the consumption weights are based on the 1985-86 National Consumer Survey. Some data on producer prices are collected, but these statistics are not comprehensive and no producer price index is compiled. As a consequence, some sectoral 
GDP deflator indices are based not on producer prices, but on consumer price subindices and ad hoc assumptions. Expenditure deflators also suffer from methodological shortcomings. There are plans to update the weighting to 1996/97 using a National Consumer Survey.

\section{Government finance}

5. Fiscal data have historically been opaque and complicated not only by the federal structure, but also by a multiplicity of off-budget funds and by accounting practices that underestimate the actual size of public expenditure. A multisector statistics mission in September 1999 noted that the most pressing shortcomings related to inadequate data coverage, the lack of monthly and quarterly compilation, and inadequate timeliness.

6. A government finance statistics (GFS) mission in early 2001 found that authorities had implemented few of the recommendations made by the 1999 multisector statistics mission. Following the creation of a National Committee on Government Finance Statistics (NCGFS), another GFS mission visited Abuja in March 2002 and found that no further progress had been made by the NCGFS, notably owing to insufficiently clear institutional arrangements and lack of sufficient staff. The mission also identified inconsistencies and difficulties of interpretation across various data sources. The mission conducted a compilation exercise using data for 2000 that showed substantial room for improvement in the consistency of source data and reconciliation between above- and below-the-line data. This exercise compiled data for the consolidated central government and general government, with special effort to capture the numerous special funds and dedicated accounts that carry out large financial transactions. The mission used the new Government Finance Statistics Manual 2001 (GFSM 2001) framework (but on a cash basis).

7. The mission also laid out a detailed 3-stage "action plan" and recommended to expand the NCGFS and increase GFS unit staffing; improve the consistency of OAGF and Central Bank of Nigeria (CBN) source data; expand CBN work to reconcile government financing data (available in-house); and have the GFS unit document an inventory of source data. Also, the action plan suggested that a second step focus on improving the coverage of reporting to include state and local government operations, as well as to ensure adequate dissemination of GFS data. In addition, a subsequent step was to focus on a more complete application of the guidelines contained in GFSM 2001.

8. A follow up mission in July 2003 found that the authorities had implemented very few of the recommendations that were put forward by the 2002 mission, including on the proposed strategy for transition to the GFSM 2001. However, the mission reported progress in specific areas, in particular in regard to the work of the CBN.

9. Annual fiscal data are not reported for inclusion in the GFS Yearbook, but aggregated fiscal data are reported for inclusion in the IFS, although no quarterly or monthly data on financing are reported. 


\section{Monetary accounts}

10. In the past, the authorities have taken steps toward implementing the key recommendations of STA money and banking technical assistance missions. However, problems remain, regarding the consistency of the interbank positions, central government deposits, and large unexplained line items within other items net. In addition, the reporting of monetary data for $I F S$ was recently interrupted because of major unresolved accounting problems at the CBN. Throughout 2006, the CBN took steps to change its core accounting system. No provision was made for a transition period in which the old and the new accounting systems would run in parallel to test the operational reliability of the new system. As a result, a number of serious distortions arose in the accounting records of the CBN, including erroneous recording of some asset balances as liabilities and vice versa, and failure to capture a number of large foreign currency transactions. The most recent data published in the STA database are those for September 2006.

\section{Balance of payments}

11. Balance of payments statistics are compiled from the foreign exchange records in the banking system and from data derived from surveys. However, oil sector data are weakened by the low response rate to survey requests from oil businesses. In the non-oil sectors, only enterprises with foreign capital participation are surveyed. The estimates of non-oil imports of goods and services are based on banking data. Private capital movements are under recorded and the trade data reported by the NBS (based on customs data) sharply differ from those reported by the $\mathrm{CBN}$ (based on banking data). Based on comparisons with counterparty data, the former are likely to significantly under-report informal trade. The 1999 STA multisector mission identified actions to be taken for improving the quality of balance of payments data. However, subsequent follow-up missions in 2000, 2005 and 2006 found that little progress had been made. After a hiatus of several years, the CBN has recently resumed submitting balance of payments data to STA; however, the balance of payments contain large negative errors of omissions, which probably reflect an underestimation of current account debit transactions. Except for some methodological changes, the compilation of balance of payments statistics continues to be severely affected by organizational weaknesses and resource constraints in the NBS, customs and the CBN. The authorities have not yet initiated compilation of international reserves data in line with the Data Template on International Reserves and Foreign Currency Liquidity. Moreover, staff has been unable to assess whether data on official reserve assets are in principle consistent with the template; the data may not be adequate for monitoring because they may not adequately reflect foreign currency liabilities or distinguish other foreign currency assets. 


\section{External debt}

12. To address problems with the data on external debt, a United Kingdom-financed technical assistance effort was launched in October 1998. After initial delays, the Debt Management Office was established in August 2000, and good progress has been made in verifying individual loan accounts with creditors' statements and improving the efficiency of debt management. A correctness and duplication audit of loan portfolio was carried out after the two separate databases of the Federal Ministry of Finance and the CBN were merged. In September 2000, the process of reconciling Nigeria's external obligations to multilateral creditors began. Following the Paris Club agreement of December 13, 2000, the verification of individual loan accounts with each individual Paris club creditor was also launched and, by October 2001, was reported to be complete. The authorities do not collect data on private sector external debt, and should work to extend the coverage of their database to include private sector liabilities. 


\section{Nigeria: Table of Common Indicators Required for Surveillance}

As of May 17, 2007

\begin{tabular}{|c|c|c|c|c|c|}
\hline & $\begin{array}{c}\text { Date of } \\
\text { latest } \\
\text { observation }\end{array}$ & $\begin{array}{l}\text { Date } \\
\text { received }\end{array}$ & $\begin{array}{c}\text { Frequency } \\
\text { of } \\
\text { Data }^{6}\end{array}$ & $\begin{array}{c}\text { Frequency } \\
\text { of } \\
\text { Reporting }^{6}\end{array}$ & $\begin{array}{c}\text { Frequency } \\
\text { of } \\
\text { publication }^{6}\end{array}$ \\
\hline Exchange Rates & Apr 2007 & May 2007 & $\mathrm{D}$ & M & $\mathrm{D}$ \\
\hline $\begin{array}{l}\text { International Reserve Assets and Reserve Liabilities of the Monetary } \\
\text { Authorities } 1\end{array}$ & Feb 2007 & Apr 2007 & M & M & M \\
\hline Reserve/Base Money & Feb 2007 & Apr 2007 & M & M & M \\
\hline Broad Money & Feb 2007 & Apr 2007 & M & $\mathrm{M}$ & $\mathrm{M}$ \\
\hline Central Bank Balance Sheet & Feb 2007 & Apr 2007 & $\mathrm{M}$ & $\mathrm{M}$ & $\mathrm{M}$ \\
\hline Consolidated Balance Sheet of the Banking System & Feb 2007 & Apr 2007 & M & M & M \\
\hline Interest Rates $^{2}$ & Apr 2007 & May 2007 & $\mathrm{D}$ & $\mathrm{D}$ & $\mathrm{D}$ \\
\hline Consumer Price Index & Mar 2007 & Apr 2007 & M & M & M \\
\hline $\begin{array}{l}\text { Revenue, Expenditure, Balance and Composition of Financing }{ }^{3}-\text { General } \\
\text { Government }\end{array}$ & Feb 2007 & Apr 2007 & M & M & M \\
\hline $\begin{array}{l}\text { Revenue, Expenditure, Balance and Composition of Financing }{ }^{3}-\text { Central } \\
\text { Government }\end{array}$ & Mar. 2007 & May 2007 & M & M & M \\
\hline Stocks of Central Government and Central Government-Guaranteed Debt ${ }^{5}$ & Dec 2006 & Feb 2007 & A & A & A \\
\hline External Current Account Balance & Dec 2006 & Feb 2007 & A & A & A \\
\hline Exports and Imports of Goods & Mar 2006 & Feb 2007 & Q & Q & A \\
\hline GDP/GNP & 2006 & Feb 2007 & A & A & A \\
\hline Gross External Debt & Dec 2006 & Feb 2007 & A & A & A \\
\hline
\end{tabular}

${ }^{1}$ Includes reserve assets pledged or otherwise encumbered as well as net derivative positions.

${ }^{2}$ Both market-based and officially-determined, including discount rates, money market rates, rates on treasury bills, notes and bonds.

${ }^{3}$ Foreign, domestic bank, and domestic nonbank financing.

${ }^{4}$ The general government consists of the central government (budgetary funds, extra budgetary funds, and social security funds) and state and local governments.

${ }^{5}$ Including currency and maturity composition.

${ }^{6}$ Daily (D), Weekly (W), Monthly (M), Quarterly (Q), Annually (A); Irregular (I); Not Available (NA) 


\section{Statement by the IMF Staff Representative \\ June 27, 2007}

1. This statement reports on developments since the staff report for the third review under the Policy Support Instrument was prepared. The thrust of the staff appraisal is not changed by these developments.

2. The monetary program remains broadly on-track. Broad money growth remained below projections, though currency issued was higher-than-expected in April and credit to the private sector rose further. Money data were provided in line with program understandings. Reconciliation of different data sources is ongoing and supported through Fund technical assistance. In May, headline inflation was 4.7 percent year-on-year and core inflation fell to 2.8 percent year-on-year.

3. The authorities undertook measures to preserve the quality of public spending through the political transition. The allocation of revenues to the states was suspended temporarily in May in advance of the handover to new state administrations. New authorizations of capital spending at the federal level were brought to a virtual standstill in April and May. Nonetheless, the staff estimates that the non-oil primary deficit of the federal government was above its April path by about 0.3 percent of non-oil GDP due to higher capital spending utilization early in the year.

4. Key economic legislation was enacted. The Fiscal Responsibility Bill was approved by both Houses and is expected to be signed into law. The Nigeria Extractive Industries Transparency Initiative (NEITI), the public procurement, the central bank, the VAT acts, among others, were signed into law.

5. Actions on taxes and fuel prices are under discussion. The outgoing administration doubled the VAT rate to 10 percent - an important step for harmonization with ECOWASand increased gasoline prices by 15 percent. In response to mounting pressures led by labor groups, the government decided to partially roll back these increases. The price of kerosene is to be reduced to its original levels, while the recent increase in gasoline prices is to be halved. The increase in VAT rates is to be rolled back.

6. Progress was made on structural reforms. The sale of 51 percent of the government's stakes in the Port Harcourt and Kaduna oil refineries (a June-2006 benchmark) was completed. As regards assessment criteria for the third review, a draft framework for consolidated supervision (December-2006 assessment criterion) was produced and is awaiting the consideration and approval of the Executive Committee on Supervision. The selection for the management contract for the Transmission Company of Nigeria has been made and the contract can be awarded once a "no objection" statement is received from the World Bank. Bids for conducting the 2006 audit of the oil and gas sector (March-2007 assessment criterion) have been received and are being reviewed.

\section{A draft National Economic Empowerment and Development Strategy for the} period 2008-2011 (NEEDS 2) was completed. The draft of this key medium-term policy document (Nigeria's PRSP) involved a broad process of consultation, and should provide useful input into designing the new government's medium-term policies. 
Press Release No. 07/146

International Monetary Fund

FOR IMMEDIATE RELEASE

Washington, D.C. 20431 USA

June 28, 2007

\section{IMF Executive Board Completes the Third Review Under the Policy Support Instrument for Nigeria}

The Executive Board of the International Monetary Fund (IMF) has completed the third review under a two-year Policy Support Instrument (PSI) for Nigeria. The PSI was approved on October 17, 2005 (see Press Release No. 05/229). The completion of this review will support Nigeria's efforts to entrench macroeconomic stability and pursue ongoing strong structural reforms.

In completing the review, on June 27, 2007, the Executive Board agreed to the waivers of nonobservance of five structural assessment criteria and two quantitative assessment criteria on reserve money and nonconcessional borrowing.

The IMF's framework for PSIs is designed for low-income countries that may not need IMF financial assistance, but still seek close cooperation with the IMF in preparation and endorsement of their policy frameworks. PSI-supported programs are based on countryowned poverty reduction strategies adopted in a participatory process involving civil society and development partners. This is intended to ensure that PSI-supported programs are consistent with a comprehensive framework for macroeconomic, structural and social policies to foster growth and reduce poverty. Members' performance under a PSI is reviewed semi-annually, irrespective of the status of the program (see Public Information Notice No. $\underline{05 / 145})$.

In commenting on the Executive Board decision, Mr. John Lipsky, First Deputy Managing Director and Acting Chair, made the following statement:

"The third scheduled review under Nigeria's Policy Support Instrument is completed. The authorities' program, which meets upper credit tranche conditionality and is guided by the National Economic Empowerment and Development Strategy, aims to balance spending needs with maintaining macroeconomic stability.

"The Nigerian authorities are to be commended for implementing their reform program in recent years. The improved policy framework, strengthened institutions and macroeconomic policies, and major progress with wide-ranging structural reforms led to impressive macroeconomic outcomes - notably robust growth and low inflation - as well as improved investor confidence. This is a strong legacy for the new government. 
"The recent performance under the program was uneven. The fiscal expansion at the consolidated government level in late 2006 resulted from a regrettable deviation from the oil price fiscal rule and led to an unanticipated liquidity injection. This did not result in excessive inflationary pressures, due largely to increased confidence and higher money demand.

"While significant progress was achieved in structural reforms over the course of the PSI, the delays in completing the final steps of several measures are regrettable. The progress in the long awaited legislative actions intended to help institutionalize the reforms under the PSI is welcome. In particular, the parliamentary passage of the Fiscal Responsibility Bill is a key step in supporting prudent management of oil revenues. Stronger bank supervision is important, including on a consolidated basis, to sustain the gains of the recent bank consolidation and recapitalization.

"The 2007 program adequately addresses the recent slippages in policy implementation by reconfirming the 2007 fiscal targets set at the second review, and maintaining a monetary program that targets single-digit inflation by partly reversing the broad money acceleration. The inflation objective should be supported by the overall fiscal contraction and a more flexible exchange rate policy going forward. Ensuring good quality of monetary data will be essential.

"Maintaining the reform momentum and safeguarding economic gains of recent years will be necessary to realize Nigeria's growth potential, which is important for Nigeria's fight against poverty. Fiscal policy should remain consistent with macroeconomic stability, and reaffirming the political agreement on the use of oil revenues among all levels of government will be important in this regard. It will be crucial to continue recent efforts to develop guidelines on the effective use of oil savings, ensure good quality of capital spending, and take forward structural reforms including trade liberalization.

"The authorities failed to ensure the accuracy of information provided to the Board on nonconcessional borrowing in the context of the completion of previous reviews under the PSI. The borrowing did not significantly undermine Nigeria's debt sustainability. In light of this, and the authorities' ongoing efforts to strengthen debt management, the Board decided to maintain a positive assessment of Nigeria's past program performance under the PSI," Mr. Lipsky said. 


\section{Statement by Peter Gakunu, Executive Director for Nigeria and Godwill Efiong Ukpong, Senior Advisor to Executive Director June 27, 2007}

1. The Nigerian authorities express their appreciation to the Fund for continuous engagement and support for their highly ambitious macroeconomic and structural reform agenda under the PSI. They particularly wish to thank management and the staff for valuable consultations, constructive dialogue, and comprehensive and informative assessment on Nigeria's economic achievements, challenges and prospects. They broadly agree with the thrust of the analyses, conclusions and recommendations in the reports.

\section{Recent Macroeconomic Developments and Performance.}

2. The strong commitment of the authorities to sustaining macroeconomic stability and implementation of structural reforms, as well as a generally favorable external environment, including Fund TA support, have underpinned the program achievements under the PSI which include: robust growth; lower inflation; a decline in debt; and accumulation of substantial reserves. Overall real GDP growth will rise from an estimated 5.2 percent in 2006 to a projected 8.2 percent in 2007 , as the corresponding slight fall in non-oil GDP from 8.9 percent to 7.5 percent is more than compensated for by the rise in oil GDP from 2.8 percent to a projected 9.9 percent. High agricultural output, induced largely by good weather conditions and supportive policies, and strong performance of communications, construction and service sectors contributed to the relatively high real GDP growth. The inflation rate, estimated at 8.2 percent in 2006, is projected to decline to 7.8 percent in 2007 in line with the single-digit inflation objective of the authorities, as monetary policy is tightened. The tempo of structural reforms has remained very high, especially regarding the speed and breadth of privatization. Government's fiscal operations, anchored on oil pricebased fiscal rule, have continued to result in overall budget surplus. Investors' confidence has risen, resulting in favorable sovereign ratings of BB-.

\section{Performance under the PSI}

3. Alongside a relatively robust macroeconomic performance the authorities have also successfully continued with the implementation of structural reforms under the PSI. In order to ensure policy continuity, the outgoing government prepared policy strategy documents, which have been handed to the new administration, which took office on May 29, 2007. The final steps in completing some of the structural measures were unavoidably delayed. Some of the delays arose from expanded scope of work related to what was originally envisaged, but most of the delayed measures have either been completed or are well advanced, and the authorities are pressing ahead toward their completion. In general, remedial measures have been taken in all cases. Notably, as assessed by staff, the missed quantitative and structural criteria do not undermine the thrust of policy framework under the PSI to which the authorities remain firmly committed. 
4. On the issue of misreporting, related to the government contracting a US\$200 million non-concessional loan, signed on January 12, 2006, the authorities agree with the concern raised by the Fund. They have, therefore responded to Management's letter on the subject and provided relevant documentation to assist clarify matters relating to the loan. As indicated in the authorities' letter to Management and in the staff report, the loan arrangement was part of a supplier's contract agreement executed on December 15, 2004 when the PSI had not come into effect and was subjected to protracted negotiations which carried through 2005 to improve its terms. The authorities have regretted the oversight in not providing information on the loan during the first and second reviews of the PSI, which was also attributable to changes in key personnel in the Ministry of Finance, poor debt database and weak debt management capacity at the time. Given this experience, the authorities have now strengthened their debt management practices.

\section{Macroeconomic Policies for 2007/08 and the Medium-term}

5. Notwithstanding the significant achievements and progress made, the Nigerian authorities recognize that many challenges still remain to further economic advancement. However, the authorities are already adopting and implementing a wide range of strong policy measures to help deal with these challenges. In this regard, a significant supply-side constraint to increased economic activity and growth, i.e. unstable electricity supply, is being addressed, as several new power plants have been brought into operation and are helping to stabilize and enhance electricity production and reduce costs. In addition, sustained actions being taken by the monetary authority, TA on Statistics, and data reconciliation are contributing to effective refinement of monetary data. Furthermore, key legislations have been enacted into law to institutionalize reforms, while a high-level national conference is being convened to address the Niger Delta problem.

6. Real GDP growth is projected to increase by 8.2 and 6.7 percent in 2007 and 2008 respectively, with rising gas production, expected recovery in oil output to 2005 levels, improved infrastructure and robust non-oil sector performance contributing to high real GDP growth. Inflationary pressures would be contained and inflation kept at single digits as the 2007 macroeconomic program targets stronger fiscal position and monetary growth that is lower than non-oil sector growth.

\section{Fiscal Policy}

7. Although the overall fiscal balance was in surplus in 2006, and allowed for further accumulation of savings, the larger-than-projected expansion in the consolidated government level largely added to domestic demand. The authorities have, therefore, reaffirmed their commitment to 2007 fiscal program (which relies on the MTEF), set during the second review. This involves a reduction in fiscal impulse, by envisaging a reduction in non-oil primary deficit of the consolidated government spending by about 3 percent of non-oil GDP. Capital spending on key infrastructure, including projects with high import content, is 
expected to increase, while other spending would be contained. Achievement of the consolidated government target would mainly depend on adherence to the oil-price fiscal rule which is already being implemented. The authorities have also prepared a comprehensive Debt Management Framework to help preserve gains made recently in debt sustainability. The DSF is providing guidance on the borrowing strategy, procedures, and acceptable terms and conditions of loans, their disposition and servicing.

\section{Monetary Policy}

8. Monetary policy partially succeeded in offsetting the unexpected fiscal stimulus of late 2006, as problems with monetary data impeded a more effective analysis of monetary conditions, thus limiting the Central Bank's ability to respond more promptly to liquidity situations. Thus, monetary aggregates grew higher than programmed. The monetary authority attributes the rise in reserve money in December 2006 to a surge in currency arising from demands associated with political campaigns, while large credits to the private sector, including for investment in the oil sector, mainly explain the rise in broad money in mid 2006. Against this background, the 2007 monetary program targets the Central Bank's single-digit inflation objective by reversing, in part, the acceleration in broad money, targeted to grow by about 12 percent (below nominal non-oil GDP growth). The Central Bank's sterilization efforts would rely on foreign exchange sales, in addition to other monetary policy instruments. The Bank would also continue to improve on prudential standards and more effective supervision to further enhance soundness of the banking and financial industry. The Central Bank of Nigeria (CBN) is refining a Financial Sector Strategy 2020 (FSS 2020) which was recently articulated for enhanced support toward further development of the Nigerian financial sector and markets.

\section{Other Structural Reforms}

9. Nigeria has significantly strengthened the management of its oil wealth in the last few years through adoption of the oil-price fiscal rule and participation in the Extractive Industries Transparency Initiative (EITI). The fiscal Responsibility and NEITI Bills have been enacted into law to institutionalize these reforms. Having states pass their respective fiscal responsibility bills is a near-term priority of government.

10. The introduction and implementation of guidelines on the use of Nigeria's oil savings and management of the economy and the policy framework through the political transition are a key challenges going forward. The authorities are committed to meeting both challenges. Indeed, they are already doing so as reputable domestic and foreign asset management institutions are involved in managing Nigerian oil savings, while the oil-price fiscal rule is now considered a welcome and transparent budget management tool. Similarly, the political transition has largely been successfully concluded, with the completion of national elections in April and inauguration on May 29, 2007 of the new President, who has reaffirmed his commitment to ongoing economic policies and reforms, improved governance, 
and fight against corruption. The new government counts on the continued support of development partners to complement its structural reform efforts.

\section{Conclusion}

The Nigerian authorities continue to be strongly committed to effective implementation of sound macroeconomic and structural reform policies to further consolidate and safeguard economic gains made under the PSI, including macro stability, high sustainable GDP growth for poverty reduction, and progress towards attainment of the MDGs. They are also committed to taking additional measures to address other challenges and risks. They welcome the Fund's engagement with the country, including through policy dialogue, advice and TA. They therefore, count on the Fund's continued support, and request for the granting of waivers for the missed quantitative and structural assessment criteria and approval for modification of the end-June 2007 monetary targets, and completion of the third review of performance under the PSI. 